\title{
Demand planning approaches to aggregating and forecasting interrelated demands for safety stock and backup capacity planning
}

\author{
ARGON CHEN*, C.-H. HSU and J. BLUE \\ Graduate Institute of Industrial Engineering, National Taiwan University, \\ 1 Roosevelt Road, Sec. 4, Taipei, Taiwan 106
}

(Revision received March 2006)

\begin{abstract}
Results of demand planning serve as the basis of every planning activity in a demand-supply network and ultimately determine the effectiveness of manufacturing and logistic planning, such as capacity and safety stock planning, in the network. The uncertainty of demand signals that are propagated and magnified over the network becomes the crucial cause of ineffective operation plans. With the globalization of demand-supply networks and the desire for a more integrated operation plan, demand planning is now one of the greatest challenges facing manufacturers. To manage the demand variability, appropriate demand aggregation and statistical forecasting approaches are known to be effective. This paper will use the bivariate VAR(1) time-series model as a study vehicle to investigate the effects of aggregating two interrelated demands. It is shown that the aggregated time series of two $\operatorname{VAR}(1)$ times series is equivalent to the sum of two AR(1) time series. Through theoretical development, the paper further explores the properties of the aggregated time series and provides guidelines for practitioners to determine proper aggregation and forecasting approaches. An important finding of this research is that demand aggregation is far more effective than statistical forecasting in operations planning for any two demands with low positive correlation or negative correlation.
\end{abstract}

Keywords: Demand planning; Demand aggregation; Demand forecast; Safety stock planning; Backup capacity planning

\section{Introduction}

Demand planning is the very first step of supply chain planning. Its results affect the quality of its subsequent planning activities. Yet, the demand signal is known to be the most unreliable information in supply chain planning. The demand uncertainty is then propagated and further magnified (Lee et al. 1997) in the supply chain. That is, the further down the supply chain level, the worse the planning quality. To improve the quality of supply chain planning, demand planning becomes one of the greatest challenges facing modern manufacturers.

It is known that demand uncertainty can be effectively reduced through appropriate demand aggregation (Caplin 1985, Simchi-Levi et al. 2000) and forecasting.

*Corresponding author. Email: achen@ntu.edu.tw; d93522042@ntu.edu.tw 


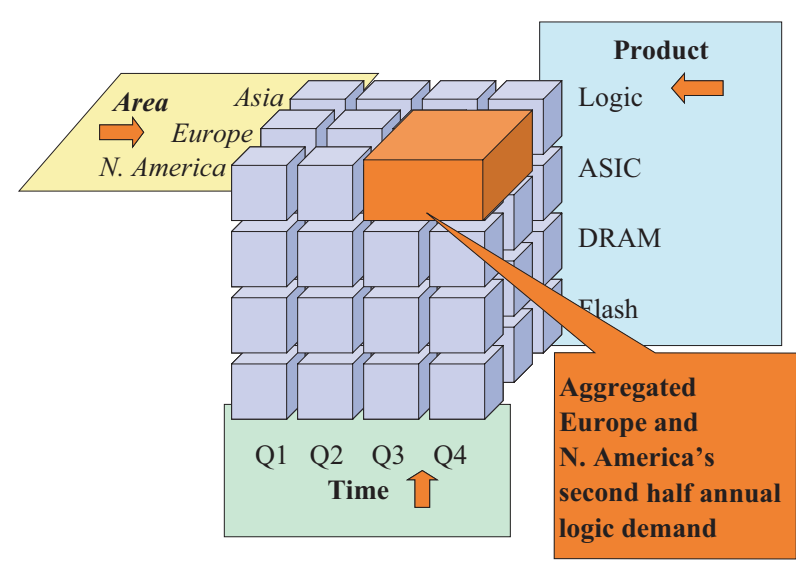

Figure 1. Aggregation and disaggregation through multiple perspectives.

An On-Line Analytical Processing (OLAP) tool is thus useful for analysis of multiperspective (multi-dimensional) demand aggregation and forecasting. Demand planners can use the tool to quickly roll up demands to an aggregated level for a total demand or drill down a total demand to detailed demands from different perspectives. For example, in figure 1, a semiconductor demand planner can roll up (or aggregate) the detailed demand to calculate the total demand for logic IC in North America and Europe during the first during the last two quarters of the year. The demand planner may find such an aggregated demand is less fluctuated and more suitable for demand forecasting and supply chain planning. The demand planner can also drill down (or disaggregate) the total demand to see, for example, the proportion of the North American market.

There are three perspectives (dimensions) of demands in figure 1: time, product type, and region. To understand better the natures of certain demands, users of OLAP tools can choose desired perspectives to perform the roll-up and/or drill-down analyses. Such analyses are also referred to as 'slice-and-dice' analyses. However, the demand planners have to rely on their own understanding of the market or simply their intuitive, subjective judgement to perform the aggregation analysis. Following demand aggregation/disaggregation, demand forecasting is the next step of demand planning also noted as an important means to improve the accuracy of demand plans. However, the effect of statistical forecasting is obscure and planners are hesitant to use the predetermined time-variant demand models because a flawed model often incurs more errors and causes poorer forecasts.

In practice, most time-variant demands are observed to follow autoregression time-series models. Particularly, the first order autoregression, AR(1), model is widely applied in both practice and literature. For example, Lau and Wang (1987) study the lead-time distribution with auto-correlated demands. Eppen and Martin (1988), Marmorstein and Zinn (1993) and Charnes et al. (1995) investigate determination of safety stock under the effects of auto-correlated demands. Lee et al. (1997) assume an AR(1) demand model for their landmark study on the supplychain bullwhip effect. Ray (1981), Fotopoulos et al. (1988) and Erkip et al. (1990) examine the ordering and safety stock strategies under correlated demands. 
However, most studies have focused on optimization of operations plans given a known demand time-series model. In contrast, our focus is on the demand planning methods and how they affect the quality of operations planning given a planning practice, namely, the safety stock or backup capacity planning.

In this paper, we will use the bivariate vector autoregression (VAR(1)) time-series model as a study vehicle to investigate the effects of aggregating two interrelated demands. In addition, throughout this paper we analyse the performance of safetystock and backup-capacity planning to illustrate how the quality of each demand planning approach affects the operations planning efficiency. To make clear the difference among demand planning approaches, we also describe each demand planning approach in terms of its use in safety stock or backup capacity planning. The goal of this paper is to use certain statistical properties of the demands to develop principles that can assist the demand planners to determine whether demand aggregation and/or statistical forecasting are needed, in particular, for safety-stock or backup-capacity planning. This paper is organized into five sections. Following the introduction section, we first briefly describe the VAR(1) demand model and five demand planning approaches. The performance of the five approaches is then analytical derived in section 3. Section 4 will use eight scenarios to evaluate and compare the performances among different approaches. Finally, principles and guidelines will be provided to practitioners for adopting appropriate aggregation/ forecasting approaches.

\section{VAR(1) demand model and demand planning approaches}

Since the interrelation of demands is the focal point of our research, the first order bivariate vector autoregression, VAR(1), time-series model (Box and Tiao 1977, Tiao and Box 1981, Tiao and Tsay 1983) is chosen as a study vehicle. Bivariate VAR(1) demands can be denoted as a vector: $\underline{X}_{t}=\left[X_{1 t}, X_{2 t}\right]^{\prime}$ and the VAR(1) model can be expressed as:

$$
\underline{X}_{t}=\underline{u}+\underline{\Phi X}_{t-1}+\underline{a}_{t}
$$

where:

$$
\begin{aligned}
& \underline{u}=\left[u_{x 1}, u_{x 2}\right]^{\prime} \text { constant vector, } \\
& \underline{\Phi}=\left[\begin{array}{ll}
\phi_{11} & \phi_{12} \\
\phi_{21} & \phi_{22}
\end{array}\right] \text { autoregression parameter matrix, } \\
& \underline{a}_{t}=\left[a_{1 t}, a_{2 t}\right]^{\prime} \quad \text { white noise vector following iid bivariate normal distribution: } \\
& N_{2}=\left(\underline{0}, \sum\right) \text { with } \\
& \underline{0}=[0,0]^{\prime}, \quad \sum=\left[\begin{array}{cc}
\sigma_{11} & 0 \\
0 & \sigma_{22}
\end{array}\right] \text {. }
\end{aligned}
$$

Model (1) can be rewritten as:

$$
\begin{aligned}
& X_{1 t}=u_{x 1}+\phi_{11} X_{1 t-1}+\phi_{12} X_{2 t-1}+a_{1 t} \\
& X_{2 t}=u_{x 2}+\phi_{21} X_{1 t-1}+\phi_{22} X_{2 t-1}+a_{2 t},
\end{aligned}
$$


where $\phi_{11}$ and $\phi_{22}$ represent the 'auto-correlation elements' that dictate how much a demand depends on its own earlier demands; and $\phi_{12}$ and $\phi_{21}$ represent the 'inter-correlation elements' that determine how the two demands correlate to each other.

It can be seen that the bivariate VAR(1) can clearly describe the interrelation of two autoregressive time series:

- When both the two interrelation element of $\underline{\Phi}, \phi_{12}$ and $\phi_{21}$, are equal to zero, the two time series are independent and can be in effect expressed as two separate AR(1) time-series models with autoregression parameters $\phi_{11}$ and $\phi_{22}$, respectively.

- When only one of $\phi_{12}$ and $\phi_{21}$ is zero, the relation will be unidirectional. That is, if $\phi_{12}=0$ and $\phi_{21} \neq 0$, then $X_{1 t}$ is a univariate $\operatorname{AR}(1)$ while $X_{2 t}$ will be affected by $X_{1 t-1}$.

- When both $\phi_{12}$ and $\phi_{21}$ are not zero, the two time series are interrelated.

- When all the elements of $\Phi, \phi_{11}, \phi_{12}, \phi_{21}$ and $\phi_{22}$, are statistically insignificant, the two time series will appear as two time-invariant data sequences.

The demand plans serve as basis of inventory planning and/or capacity planning. Safety stock and/or backup capacity are prepared to minimize the effect of demand uncertainty. For instance, under the $(s, S)$ inventory policy, the reorder point $s$ is set based on a safety stock level that meets a predetermined service level (Caplin 1985, Silver et al. 1998):

$$
s=L \times A V G+z \times S T D \times \sqrt{L},
$$

where:

$A V G$ average unit time demand,

$S T D$ standard deviation of unit time demand,

$\begin{aligned} z \times S T D \times \sqrt{L} \quad & \text { replenishment lead time, } \\ & \text { safety stock prepared to meet a desired service level under demand } \\ & \text { uncertainty. }\end{aligned}$

It can be seen that the inventory cost under the $(s, S)$ policy increases as the demand uncertainty, i.e. STD, grows. Similarly, the capacity cost rises owing to the preparation of backup capacity for the uncertain demand. However, demands are not aggregated without an additional planning cost. In the inventory problem, the replenishment lead-time is often prolonged because the inventory planned for the aggregated demand is stocked in a centralized warehouse and the transportation distance to the demand sources is thus extended. For the capacity planning problem, aggregating demands often means a higher product mix on the shop floor and causes possibly lower yield, more machine change-over time and higher machine breakdown rate.

In this research, we investigate five possible demand-planning approaches in response to two interrelated demands as follows:

- Approach 1: the manufacturer lacks the technology of statistical forecasting. Demands are handled as simple time-invariant data sequences. 
The demand variability is measured by the standard deviation. The safety stock (or backup capacity) is planned separately for each demand based on a multiple of its standard deviation.

- Approach 2: the manufacturer aggregates the two demands together. The aggregated demand, denoted by $Y_{t}\left(=X_{1 t}+X_{2 t}\right)$, is handled as a time-invariant data sequence. The safety stock (or backup capacity) is planned for two demands together based on a multiple of $Y_{t}$ 's standard deviation.

- Approach 3: the manufacturer owns the statistical forecasting technology but lacks knowledge of multivariate time series. Demands are handled as two independent time series. AR(1) time-series models are used as the statistical forecasting models. Statistical forecasting is carried out separately based on the estimated AR(1) time-series model for each demand. The safety stock (or backup capacity) is planned separately for each demand based on a multiple of its forecast standard error.

- Approach 4: the manufacturer aggregates the two demands together. The aggregated demand is handled as an $\operatorname{AR}(1)$ time series. The safety stock (or backup capacity) is planned for two demands together based on a multiple of the $Y_{t}$ 's forecast standard error.

- Approach 5: the manufacturer owns the technology of forecasting multivariate time series. Statistical forecasting is based on the VAR(1) model. That is, the company is able to consider the interrelationship between two demands in their forecast model. The forecast errors for individual demands are estimated from the common VAR(1) model and the safety stock (and/or backup capacity) is planned individually for each demand based on a multiple of its forecast standard error.

The five different approaches are summarized and compared in table 1.

It should be noted that the fourth approach aggregates the two demands first before a univariate time-variant model, $\mathrm{AR}(1)$, is applied to make forecast for the aggregated demand while the fifth approach makes forecasts for the two demands simultaneously using a multivariate time-variant model, $\operatorname{VAR}(1)$.

\section{Theoretical development of demand planning performance}

Though the demands are actually from an underlying time-variant VAR(1) model, Approaches 1 and 2 do not make statistical forecasts based on demands' time-variant nature. Rather, these two approaches directly use the standard deviations of demands as the bases for safety stock or backup capacity planning. We first derive the theoretical variances, $\sigma_{x 1 x 1}$ and $\sigma_{x 2 x 2}$, for the two $\operatorname{VAR}(1)$ time series, $X_{1 t}$ and $X_{2 t}$, respectively. Without loss of generality, we assume that the constants are zero, i.e. $\underline{u}=\underline{0}$ in Model (1):

$$
\underline{X}_{t}=\underline{\Phi X}_{t-1}+\underline{a}_{t}
$$


Table 1. Summary of the demand planning approaches.

\begin{tabular}{|c|c|c|c|c|c|c|c|}
\hline \multicolumn{3}{|c|}{$\begin{array}{l}\text { Time-variant } \\
\text { forecast }\end{array}$} & \multicolumn{2}{|c|}{$\begin{array}{c}\text { Multivariate } \\
\text { view }\end{array}$} & \multicolumn{2}{|c|}{$\begin{array}{l}\text { Aggregate } \\
\text { to forecast }\end{array}$} & \multirow[b]{2}{*}{ Explanations } \\
\hline & Yes & No & Yes & No & Yes & No & \\
\hline 1 & & $\sqrt{ }$ & & $\sqrt{ }$ & & $\sqrt{ }$ & $\begin{array}{l}X_{1 t} \text { and } X_{2 t} \text { are handled as two time- } \\
\text { invariant demands and are predicted } \\
\text { based only on their respective variations }\end{array}$ \\
\hline 2 & & $\sqrt{ }$ & & $\sqrt{ }$ & $\sqrt{ }$ & & $\begin{array}{l}Y_{t}\left(=X_{1 t}+X_{2 t}\right) \text { is handled as a time-invariant } \\
\text { demand and forecasted based only on } \\
\text { its variation }\end{array}$ \\
\hline 3 & $\sqrt{ }$ & & & $\sqrt{ }$ & & $\sqrt{ }$ & $\begin{array}{l}X_{1 t} \text { and } X_{2 t} \text { are handled as two time-variant } \\
\text { demands and predicted using two } \\
\text { respective AR(1) models }\end{array}$ \\
\hline 4 & $\sqrt{ }$ & & & $\sqrt{ }$ & $\sqrt{ }$ & & $\begin{array}{l}Y_{t}\left(=X_{1 t}+X_{2 t}\right) \text { is handled as a time-variant } \\
\text { demand and predicted using an } \\
\mathrm{AR}(1) \text { model }\end{array}$ \\
\hline 5 & $\sqrt{ }$ & & $\sqrt{ }$ & & & $\sqrt{ }$ & $\begin{array}{l}X_{1 t} \text { and } X_{2 t} \text { are handled as bivariate } \\
\text { demands and predicted using a } \\
\text { VAR(1) model }\end{array}$ \\
\hline
\end{tabular}

One can then obtain $\sigma_{x 1 x 1}$ and $\sigma_{x 2 x 2}$ as follows (for a derivation, see appendix 1):

$$
\begin{aligned}
\sigma_{x 1 x 1}= & -\left(\phi_{12}^{2} \sigma_{22}-\phi_{21} \sigma_{11} \phi_{12}-\phi_{22} \sigma_{11} \phi_{11}-\sigma_{22} \phi_{21} \phi_{12}^{3}-\phi_{22}^{2} \sigma_{11}+\phi_{22}^{3} \sigma_{11} \phi_{11}\right. \\
& \left.+\phi_{22} \phi_{12}^{2} \phi_{11} \sigma_{22}-\phi_{21} \phi_{22}^{2} \sigma_{11} \phi_{12}+\sigma_{11}\right) /\left(\phi_{11}^{2}-\phi_{11} \phi_{22}^{3}+\phi_{21} \phi_{22}^{2} \phi_{12}+\phi_{21} \phi_{11}^{2} \phi_{12}\right. \\
& +\phi_{11}^{3} \phi_{22}^{3}-\phi_{11}^{3} \phi_{22}-3 \phi_{21} \phi_{11}^{2} \phi_{12} \phi_{22}^{2}+3 \phi_{21}^{2} \phi_{11} \phi_{12}^{2} \phi_{22}+\phi_{21} \phi_{12}+\phi_{21}^{2} \phi_{12}^{2} \\
& \left.+\phi_{22} \phi_{11}-1-\phi_{21}^{3} \phi_{12}^{3}+\phi_{22}^{2}-\phi_{11}^{2} \phi_{22}^{2}\right) \\
\sigma_{x 2 x 2}= & -\left(\phi_{11}^{3} \phi_{22} \sigma_{22}+\phi_{22} \phi_{21}^{2} \sigma_{11} \phi_{11}-\phi_{22} \phi_{11} \sigma_{22}-\phi_{11}^{2} \sigma_{22}-\phi_{21} \phi_{11}^{2} \phi_{12} \sigma_{22}-\phi_{21}^{3} \sigma_{11} \phi_{12}\right. \\
& \left.+\phi_{21}^{2} \sigma_{11}+\sigma_{22}-\sigma_{22} \phi_{21} \phi_{12}\right) /\left(\phi_{11}^{2}-\phi_{11} \phi_{22}^{3}+\phi_{21} \phi_{22}^{2} \phi_{12}+\phi_{21} \phi_{11}^{2} \phi_{12}+\phi_{11}^{3} \phi_{22}^{3}\right. \\
& -\phi_{11}^{3} \phi_{22}-3 \phi_{21} \phi_{11}^{2} \phi_{12} \phi_{22}^{2}+3 \phi_{21}^{2} \phi_{11} \phi_{12}^{2} \phi_{22}+\phi_{21} \phi_{12}+\phi_{21}^{2} \phi_{12}^{2}+\phi_{22} \phi_{11} \\
& \left.-1-\phi_{21}^{3} \phi_{12}^{3}+\phi_{22}^{2}-\phi_{11}^{2} \phi_{22}^{2}\right)
\end{aligned}
$$

Equations (4) and (5) are theoretical variances of the two demands under the VAR(1) model. Suppose Approach 1 uses an unbiased estimator, such as the sample variance statistic, to estimate the variances. The expected safety stock (or expected backup capacity), prepared under Approach 1, can be found by (2) to base on a multiple of $\sigma_{x 1}\left(=\sqrt{\sigma_{x 1 x 1}}\right)$ and a multiple of $\sigma_{x 2}\left(=\sqrt{\sigma_{x 2 x 2}}\right)$ for individual demand series $X_{1 t}$ and $X_{2 t}$, respectively. Then, the total expected safety stock (or total expected backup capacity) prepared under Approach 1 would be based on a multiple of $\left(\sigma_{x 1}+\sigma_{x 2}\right)$. 
We can also derive the theoretical variance of the aggregated demand series $Y_{t}=X_{1 t}+X_{2 t}$ under the $\operatorname{VAR}(1)$ model:

$$
\sigma_{y y}=\sigma_{x 1 x 1}+\sigma_{x 2 x 2}+2 \sigma_{x 1 x 2}
$$

where (see also appendix 1):

$$
\begin{aligned}
\sigma_{x 1 \times 2}= & \left(\phi_{21} \sigma_{11} \phi_{11} \phi_{22}^{2}-\phi_{21}^{2} \sigma_{11} \phi_{22} \phi_{12}+\phi_{11}^{2} \sigma_{22} \phi_{22} \phi_{12}-\phi_{21} \sigma_{11} \phi_{11}-\sigma_{22} \phi_{22} \phi_{12}\right. \\
& \left.-\phi_{21} \phi_{12}^{2} \phi_{11} \sigma_{22}\right) /\left(\phi_{11}^{2}-\phi_{11} \phi_{22}^{3}+\phi_{21} \phi_{22}^{2} \phi_{12}+\phi_{21} \phi_{11}^{2} \phi_{12}+\phi_{11}^{3} \phi_{22}^{3}-\phi_{11}^{3} \phi_{22}\right. \\
& -3 \phi_{21} \phi_{11}^{2} \phi_{12} \phi_{22}^{2}+3 \phi_{21}^{2} \phi_{11} \phi_{12}^{2} \phi_{22}+\phi_{21} \phi_{12}+\phi_{21}^{2} \phi_{12}^{2}+\phi_{22} \phi_{11}-1-\phi_{21}^{3} \phi_{12}^{3} \\
& \left.+\phi_{22}^{2}-\phi_{11}^{2} \phi_{22}^{2}\right) .
\end{aligned}
$$

Similarly, when an unbiased estimator is used by Approach 2 to estimate the variance of the aggregated demand series, the expected safety stock (or expected backup capacity) prepared under Approach 2 would be based on a multiple of the aggregated standard deviation $\sigma_{y}=\sqrt{\sigma_{y y}}$.

To derive the forecast standard error under Approach 3, where two demands are handled separately with two individual time-series models, we need to know first how the individual demand time series looks like under the VAR(1) model. The answer lies in Theorem 1.

Theorem 1: If $X_{1 t}$ and $X_{2 t}$ follow VAR(1) model in (2), then $X_{1 t}$ can be expressed as $V_{1 t}+V_{2 t}$ where $V_{1 t}$ and $V_{2 t}$ are two $A R(1)$ time series:

$$
\begin{aligned}
& V_{1 t}=\lambda_{1} V_{1 t-1}+a_{1 t}^{v} \quad \text { and } \quad a_{1 t}^{v}=\frac{e_{11} e_{22}}{e_{11} e_{22}-e_{12} e_{21}} a_{1 t}-\frac{e_{11} e_{21}}{e_{11} e_{22}-e_{12} e_{21}} a_{2 t} \\
& V_{2 t}=\lambda_{2} V_{2 t-1}+a_{2 t}^{v} \quad \text { and } \quad a_{2 t}^{v}=\frac{-e_{21} e_{12}}{e_{11} e_{22}-e_{12} e_{21}} a_{1 t}+\frac{e_{21} e_{11}}{e_{11} e_{22}-e_{12} e_{21}} a_{2 t} .
\end{aligned}
$$

Similarly, $X_{2 t}$ can be expressed as $W_{1 t}+W_{2 t}$ where $W_{1 t}$ and $W_{2 t}$ are two $A R(1)$ time series:

$$
\begin{aligned}
& W_{1 t}=\lambda_{1} W_{1 t-1}+a_{1 t}^{w} \quad \text { and } \quad a_{1 t}^{w}=\frac{e_{12} e_{22}}{e_{11} e_{22}-e_{12} e_{21}} a_{1 t}-\frac{e_{12} e_{21}}{e_{11} e_{22}-e_{12} e_{21}} a_{2 t} \\
& W_{2 t}=\lambda_{2} W_{2 t-1}+a_{2 t}^{w} \quad \text { and } \quad a_{2 t}^{w}=\frac{-e_{22} e_{12}}{e_{11} e_{22}-e_{12} e_{21}} a_{1 t}+\frac{e_{22} e_{11}}{e_{11} e_{22}-e_{12} e_{21}} a_{2 t}
\end{aligned}
$$

In Equations (7) and (8), $\lambda_{1}$ and $\lambda_{2}$ are two eigenvalues of $\Phi$ with corresponding eigenvectors:

$$
\underline{\mathrm{e}}_{1}=\left[\begin{array}{l}
e_{11} \\
e_{12}
\end{array}\right] \quad \text { and } \quad \underline{\mathrm{e}}_{2}=\left[\begin{array}{l}
e_{21} \\
e_{22}
\end{array}\right] .
$$

The proof of Theorem 1 is shown in appendix 2 .

The result of Theorem 1 indicates that when the two time series under VAR(1) model are observed separately, each of them emerges to be a time series resembled 
by the sum of two AR(1) time series. Granger and Morris (1976) have proved that the sum of two ARMA time series will be also an ARMA time series (also Singh and Nirmalan 1989). For demand planning practice as in Approach 3, AR(1) model is usually used as the demand time-series model. That is, though the individual time series under VAR(1) model is actually the sum of two AR(1) series, demand planners in Approach 3 would still treat it as an AR(1) time series. Suppose now that maximum likelihood estimators (MLE) is used for estimation of the thought-to-be AR(1) model. Theorem 2 is then to help us estimate the forecast standard error when the sum of two AR(1) time series is estimated as an $\mathrm{AR}(1)$ time series.

Theorem 2: $D_{1 t}$ and $D_{2 t}$ are two stationary $A R(1)$ time series:

$$
\begin{aligned}
& D_{1 t}=u_{D 1}+\varphi_{1} D_{1 t-1}+\varepsilon_{1 t} \quad \text { and } \quad \varepsilon_{1 t} \stackrel{\text { i.i.d. }}{\sim} N\left(0, \sigma_{\varepsilon 1}^{2}\right) \\
& D_{2 t}=u_{D 2}+\varphi_{2} D_{2 t-1}+\varepsilon_{2 t} \quad \text { and } \quad \varepsilon_{2 t} \stackrel{\text { i.i.d. }}{\sim} N\left(0, \sigma_{\varepsilon 2}^{2}\right) .
\end{aligned}
$$

Let $D_{t}$ be the sum of $D_{1 t}$ and $D_{2 t}$, i.e. $D_{t}=D_{1 t}+D_{2 t}$. Suppose that $D_{t}$ is thought to be an $A R(1)$ time series, i.e. $D_{t}=u_{D}+\varphi_{a} D_{t-1}+\varepsilon_{t}$, where $\varepsilon_{t} \stackrel{\text { i.i.d. }}{\sim} N\left(0, \sigma_{\varepsilon}^{2}\right)$. If $\varphi_{a}$ and $\sigma_{\varepsilon}^{2}$ are estimated using MLE, then their expected values can be found to be:

$$
\begin{aligned}
& E\left(\hat{\varphi}_{a}\right)=\frac{\left[\varphi_{1} \sigma_{D 1 D 1}+\sigma_{D 1 D 2}(1)+\sigma_{D 2 D 1}(1)+\varphi_{2} \sigma_{D 2 D 2}\right]+\left[\mu_{D 1}+\mu_{D 2}\right]^{2}}{\left[\sigma_{D 1 D 1}+2 \sigma_{D 1 D 2}+\sigma_{D 2 D 2}\right]+\left[\mu_{D 1}+\mu_{D 2}\right]^{2}} \\
& E\left(\hat{\sigma}_{\varepsilon}^{2}\right)=\left[\sigma_{D 1 D 1}+2 \sigma_{D 1 D 2}+\sigma_{D 2 D 2}+\left(\mu_{D 1}+\mu_{D 2}\right)^{2}\right]\left[1-E\left(\hat{\varphi}_{a}\right)^{2}\right],
\end{aligned}
$$

where:

$\mu_{D 1}$ mean of time series $D_{1 t}$,

$\mu_{D 2}$ mean of time series $D_{2 t}$,

$\sigma_{D 1 D 2}(1)$ covariance of $D_{1 t}$ and $D_{2(t-1)}$ for any $t$,

$\sigma_{D 2 D 1}(1)$ covariance of $D_{2 t}$ and $D_{1(t-1)}$ for any $t$,

$\sigma_{D 2 D 1}$ covariance of $D_{2 t}$ and $D_{1 t}$ for any $t$.

The proof is shown in appendix 3 .

Based on Theorem 1, the two demand time series $X_{1 t}$ and $X_{2 t}$ can be expressed as $X_{1 t}=V_{1 t}+V_{2 t}$ and $X_{2 t}=W_{1 t}+W_{2 t}$, respectively. Under Approach 3, the two time series are estimated as two separated AR(1) time series as follows:

$$
\begin{aligned}
& X_{1 t}=\varphi_{a}^{x_{1}} X_{1 t-1}+a_{t}^{x_{1}} \\
& X_{2 t}=\varphi_{a}^{x_{2}} X_{2 t-1}+a_{t}^{x_{2}} .
\end{aligned}
$$

From Theorem 2, when MLE is used for model estimation, the forecast meansquared errors incurred by Approach are calculated as follows:

$$
\begin{gathered}
E\left(\hat{\sigma}_{a_{t}^{x_{1}}}^{2}\right)=\left[\sigma_{V 1}^{2}+2 \sigma_{V 1 V 2}+\sigma_{V 2}^{2}+\left(\mu_{V 1}+\mu_{V 2}\right)^{2}\right]\left[1-E\left(\hat{\varphi}_{a}^{x_{1}}\right)^{2}\right] \\
E\left(\hat{\sigma}_{a_{t}^{x_{2}}}^{2}\right)=\left[\sigma_{W 1}^{2}+2 \sigma_{W 1 W 2}+\sigma_{W 2}^{2}+\left(\mu_{W 1}+\mu_{W 2}\right)^{2}\right]\left[1-E\left(\hat{\varphi}_{a}^{x_{2}}\right)^{2}\right],
\end{gathered}
$$


where:

$$
\begin{aligned}
E\left(\hat{\varphi}_{a}^{x_{1}}\right) & =\frac{\left[\lambda_{1} \sigma_{V 1}^{2}+\sigma_{V 1 V 2}(1)+\sigma_{V 2 V 1}(1)+\lambda_{2} \sigma_{V 2}^{2}\right]+\left[\mu_{V 1}+\mu_{V 2}\right]^{2}}{\left[\sigma_{V 1}^{2}+2 \sigma_{V 1 V 2}+\sigma_{V 2}^{2}\right]+\left[\mu_{V 1}+\mu_{V 2}\right]^{2}} ; \\
E\left(\hat{\varphi}_{a}^{x_{2}}\right) & =\frac{\left[\lambda_{1} \sigma_{W 1}^{2}+\sigma_{W 1 W 2}(1)+\sigma_{W 2 W 1}(1)+\lambda_{2} \sigma_{W 2}^{2}\right]+\left[\mu_{W 1}+\mu_{W 2}\right]^{2}}{\left[\sigma_{W 1}^{2}+2 \sigma_{W 1}{ }^{2}+\sigma_{W 2}^{2}\right]+\left[\mu_{W 1}+\mu_{W 2}\right]^{2}} ; \\
\sigma_{V 1 V 2} & =\frac{-\left[\left(e_{11} e_{22} e_{21} e_{12} / C^{2}\right) \sigma_{11}+\left(e_{11} e_{21} / C\right)^{2} \sigma_{22}\right]}{1-\lambda_{1} \lambda_{2}} ; \\
\sigma_{W 1 W 2} & =\frac{-\left[\left(e_{12} e_{22} / C\right)^{2} \sigma_{11}+\left(e_{11} e_{22} e_{21} e_{12} / C^{2}\right) \sigma_{22}\right]}{1-\lambda_{1} \lambda_{2}} ; \\
\sigma_{V 1 V 2}(1) & =\lambda \sigma_{V 1 V 2} ; \sigma_{V 2 V 1}(1)=\lambda_{2} \sigma_{V 1 V 2} ; \sigma_{W 1 W 2}(1)=\lambda_{1} \sigma_{W 1 W 2} ; \sigma_{W 2 W 1}(1)=\lambda_{2} \sigma_{W 1 W 2} ; \\
\sigma_{V 1}^{2} & =\frac{\sigma_{a_{1}^{v}}^{2}}{1-\lambda_{1}^{2}} ; \sigma_{a_{1}^{v}}^{2}=\left(\frac{e_{11} e_{22}}{C}\right)^{2} \sigma_{11}+\left(\frac{e_{11} e_{21}}{C}\right)^{2} \sigma_{22} ; \\
\sigma_{V 2}^{2} & =\frac{\sigma_{a_{2}^{v}}^{2}}{1-\lambda_{2}^{2}} ; \sigma_{a_{2}^{v}}^{2}=\left(\frac{e_{21} e_{12}}{C}\right)^{2} \sigma_{11}+\left(\frac{e_{21} e_{11}}{C}\right)^{2} \sigma_{22} ; \\
\sigma_{W 1}^{2} & =\frac{\sigma_{a_{1}^{w}}^{2}}{1-\lambda_{1}^{2}} ; \sigma_{a_{1}^{w}}^{2}=\left(\frac{e_{12} e_{22}}{C}\right)^{2} \sigma_{11}+\left(\frac{e_{12} e_{21}}{C}\right)^{2} \sigma_{22} ; \\
\sigma_{W 2}^{2} & =\frac{\sigma_{a_{2}^{w}}^{2}}{1-\lambda_{2}^{2}} ; \sigma_{a_{2}^{w}}^{2}=\left(\frac{e_{22} e_{12}}{C}\right)^{2} \sigma_{11}+\left(\frac{e_{22} e_{11}}{C}\right)^{2} \sigma_{22} ; \text { and } \\
C & =e_{11} e_{22}-e_{12} e_{21} .
\end{aligned}
$$

Approach 3 prepares the safety stock (or backup capacity) for each demand separately based on a multiple of the individual AR(1) forecast error. The total safety stock prepared can be then calculated based on a multiple of the sum of forecast standard errors, $\sqrt{E\left(\hat{\sigma}_{a_{t}^{x_{1}}}^{2}\right)}+\sqrt{E\left(\hat{\sigma}_{a_{t}^{x_{1}}}^{2}\right)}$.

To derive the forecast standard error for Approach 4, where two demand series obeying the VAR(1) model are aggregated, we need to know first how this aggregated time series resembles. Corollary 1 gives us the answer.

Corollary 1: $X_{1 t}$ and $X_{2 t}$ follow the VAR(1) model in (2). If $Y_{t}$ is the sum of $X_{1 t}$ and $X_{2 t}$, then $Y_{t}$ can be expressed as $U_{1 t}+U_{2 t}$ where $U_{1 t}$ and $U_{2 t}$ are two $A R(1)$ time series:

$$
\begin{aligned}
& U_{1 t}=\lambda_{1} U_{1 t-1}+a_{1 t}^{u} \text { and } a_{1 t}^{u}=\left(\frac{e_{12}+e_{11}}{e_{11} e_{22}-e_{12} e_{21}}\right)\left(e_{22} a_{1 t}-e_{21} a_{2 t}\right) \\
& U_{2 t}=\lambda_{2} U_{2 t-1}+a_{2 t}^{u} \text { and } a_{2 t}^{u}=\left(\frac{e_{22}+e_{21}}{e_{11} e_{22}-e_{12} e_{21}}\right)\left(-e_{12} a_{1 t}+e_{11} a_{2 t}\right) .
\end{aligned}
$$

The proof is shown in appendix 4.

Corollary 1 shows that the time series aggregated from two VAR(1) time series is again a sum of two $\mathrm{AR}(1)$ time series. It is supposed again $\mathrm{AR}(1)$ model is used for forecasting the aggregated time series in Approach 4:

$$
Y_{t}=\varphi_{a}^{y} Y_{t-1}+a_{t}^{y} .
$$


Results of Theorem 2 can be again applied to calculate the forecast mean-squared error for Approach 4:

$$
E\left(\hat{\sigma}_{a_{t}^{y}}^{2}\right)=\left[\sigma_{U 1}^{2}+2 \sigma_{U 1 U 2}+\sigma_{U 2}^{2}+\left(\mu_{U 1}+\mu_{U 2}\right)^{2}\right]\left[1-E\left(\hat{\varphi}_{a}^{y}\right)^{2}\right],
$$

where:

$$
\begin{aligned}
E\left(\hat{\varphi}_{a}^{y}\right)= & \frac{\left[\lambda_{1} \sigma_{U 1}^{2}+\sigma_{U 1 U 2}(1)+\sigma_{U 2 U 1}(1)+\lambda_{2} \sigma_{U 2}^{2}\right]+\left[\mu_{U 1}+\mu_{U 2}\right]^{2}}{\left[\sigma_{U 1}^{2}+2 \sigma_{U 1 U 2}+\sigma_{U 2}^{2}\right]+\left[\mu_{U 1}+\mu_{U 2}\right]^{2}} ; \\
\sigma_{U 1 U 2}= & \frac{1}{\lambda_{1} \lambda_{2}-1}\left\{\left(\frac{e_{12}^{2} e_{22}^{2}+e_{12}^{2} e_{21} e_{22}+e_{22}^{2} e_{11} e_{12}+e_{11} e_{22} e_{21} e_{12}}{C^{2}}\right) \sigma_{11}\right. \\
& \left.+\left(\frac{e_{12} e_{21} e_{22} e_{11}+e_{21}^{2} e_{11} e_{12}+e_{11}^{2} e_{21} e_{22}+e_{11}^{2} e_{21}^{2}}{C}\right)^{2} \sigma_{22}\right\} \\
\sigma_{U 1 U 2}(1)= & \lambda_{1} \sigma_{U 1 U 2} ; \quad \sigma_{U 2 U}(1)=\lambda_{2} \sigma_{U 1 U 2} \\
\sigma_{U 1}^{2}= & \frac{\sigma_{a_{1}^{u}}^{2}}{1-\lambda_{1}^{2}} ; \quad \sigma_{a_{1}^{u}}^{2}=\left(\frac{\left(e_{12}+e_{11}\right) e_{22}}{C}\right)^{2} \sigma_{11}+\left(\frac{\left(e_{12}+e_{11}\right) e_{21}}{C}\right)^{2} \sigma_{22} ; \\
\sigma_{U 2}^{2}= & \frac{\sigma_{a_{2}^{u}}^{2}}{1-\lambda_{2}^{2}} ; \quad \sigma_{a_{2}^{u}}^{2}=\left(\frac{\left(e_{22}+e_{21}\right) e_{12}}{C}\right)^{2} \sigma_{11}+\left(\frac{\left(e_{22}+e_{21}\right) e_{11}}{C}\right)^{2} \sigma_{22} ; \text { and } \\
C= & e_{11} e_{22}-e_{12} e_{21} .
\end{aligned}
$$

Thus, under Approach 4 the safety stock (or backup capacity) is prepared based on a multiple of the aggregated forecast standard error, $\sqrt{E\left(\hat{\sigma}_{a_{t}^{y}}^{2}\right)}$.

For Approach 5, forecast standard error will be simply the standard deviations of white noises: $\sqrt{\sigma_{11}}$ and $\sqrt{\sigma_{22}}$, since the correct VAR(1) model will be used for the statistical forecast and only the white noises are unpredictable and will be let out by the forecast. The safety stock (or backup capacity) will be prepared for each demand separately. The total safety stock preparation will be then based on the sum of the two forecast standard errors, $\sqrt{\sigma_{11}}+\sqrt{\sigma_{22}}$.

\section{Evaluation of demand planning approaches}

Since the inventory or capacity cost is proportional to the demand uncertainty or the forecast error, the cost will be reduced when the demand is more certain or the forecast is more accurate. Approach 1 is the most primitive demand planning approach where both the aggregation and statistical forecast are not available. In this approach, only the uncertainty of individual demands, measured by standard deviations, will be used to prepare the safety stock or backup capacity. To evaluate the performance of Approaches 2-5, we compare each approach's inventory (or capacity) cost against the inventory (or capacity) cost under Approach 1:

(1) Cost reduction of Approach 2:

$$
\mathrm{CR} 2=\frac{\text { Approach } 1 \text { cost }- \text { Approach } 2 \text { cost }}{\text { Approach } 1 \text { cost }}=\frac{\left(\sigma_{x 1}+\sigma_{x 2}\right)-\sigma_{y}}{\sigma_{x 1}+\sigma_{x 2}} .
$$


(2) Cost reduction of Approach 3:

$\mathrm{CR} 3=\frac{\text { Approach } 1 \text { cost }- \text { Approach } 3 \operatorname{cost}}{\text { Approach } 1 \text { cost }}=\frac{\left(\sigma_{x 1}+\sigma_{x 2}\right)-\left[\sqrt{E\left(\hat{\sigma}_{a_{t}^{x_{1}}}^{2}\right)}+\sqrt{E\left(\hat{\sigma}_{a_{t}^{x_{2}}}^{2}\right)}\right]}{\sigma_{x 1}+\sigma_{x 2}}$.

(3) Cost reduction of Approach 4:

$$
\mathrm{CR} 4=\frac{\text { Approach } 1 \text { cost }- \text { Approach } 4 \text { cost }}{\text { Approach } 1 \text { cost }}=\frac{\left(\sigma_{x 1}+\sigma_{x 2}\right)-\sqrt{E\left(\hat{\sigma}_{a_{t}^{y}}^{2}\right)}}{\sigma_{x 1}+\sigma_{x 2}} .
$$

(4) Cost reduction of Approach 5:

$$
\mathrm{CR} 5=\frac{\text { Approach } 1 \text { cost }- \text { Approach } 5 \text { cost }}{\text { Approach } 1 \text { cost }}=\frac{\left(\sigma_{x 1}+\sigma_{x 2}\right)-\left(\sqrt{\sigma_{11}}+\sqrt{\sigma_{22}}\right)}{\sigma_{x 1}+\sigma_{x 2}} .
$$

To evaluate the performances for different situations where the relationship between two demands varies, we design evaluation scenarios that are typical and comprehensive. The relationship between two demands can be characterized by the signs of the elements in $\underline{\Phi}$ of (2). There are 16 possible scenarios:

Scenarios 1-8:

$$
\begin{aligned}
& {\left[\begin{array}{ll}
+ & + \\
+ & +
\end{array}\right]\left[\begin{array}{ll}
+ & - \\
+ & +
\end{array}\right]\left[\begin{array}{ll}
+ & + \\
- & +
\end{array}\right]\left[\begin{array}{ll}
+ & + \\
+ & -
\end{array}\right]\left[\begin{array}{ll}
+ & - \\
- & +
\end{array}\right]\left[\begin{array}{ll}
+ & - \\
+ & -
\end{array}\right]} \\
& {\left[\begin{array}{ll}
+ & + \\
- & -
\end{array}\right]\left[\begin{array}{ll}
+ & - \\
- & -
\end{array}\right] .}
\end{aligned}
$$

Scenarios 9-16:

$$
\begin{aligned}
& {\left[\begin{array}{ll}
- & - \\
- & -
\end{array}\right]\left[\begin{array}{ll}
- & + \\
- & -
\end{array}\right]\left[\begin{array}{ll}
- & - \\
+ & -
\end{array}\right]\left[\begin{array}{ll}
- & - \\
- & +
\end{array}\right]\left[\begin{array}{ll}
- & + \\
+ & -
\end{array}\right]\left[\begin{array}{ll}
- & + \\
- & +
\end{array}\right]} \\
& {\left[\begin{array}{ll}
- & - \\
+ & +
\end{array}\right]\left[\begin{array}{ll}
- & + \\
+ & +
\end{array}\right]}
\end{aligned}
$$

Since Scenarios 9-16 are symmetrical to scenarios $1-8$, we need only the first eight scenarios for analysis. Thus, the following eight scenarios are established and used in our analyses:

Scenario 1: $\quad$ Scenario 2: $\quad$ Scenario 3: $\quad$ Scenario 4:

$\underline{\Phi}=\left[\begin{array}{ll}+0.4 & +0.3 \\ +0.3 & +0.4\end{array}\right] \quad \underline{\Phi}=\left[\begin{array}{ll}+0.4 & -0.3 \\ +0.3 & +0.4\end{array}\right] \quad \underline{\Phi}=\left[\begin{array}{cc}+0.4 & +0.3 \\ -0.3 & +0.4\end{array}\right] \quad \underline{\Phi}=\left[\begin{array}{ll}+0.4 & +0.3 \\ +0.3 & -0.4\end{array}\right]$

Scenario 5: $\quad$ Scenario 6: $\quad$ Scenario 7: $\quad$ Scenario 8:

$\underline{\Phi}=\left[\begin{array}{ll}+0.4 & -0.3 \\ -0.3 & +0.4\end{array}\right] \quad \underline{\Phi}=\left[\begin{array}{ll}+0.4 & -0.3 \\ +0.3 & -0.4\end{array}\right] \quad \underline{\Phi}=\left[\begin{array}{cc}+0.4 & +0.3 \\ -0.3 & -0.4\end{array}\right] \quad \underline{\Phi}=\left[\begin{array}{cc}+0.4 & -0.3 \\ -0.3 & -0.4\end{array}\right]$. 
In addition to $\Phi$, the ratio between the standard deviations of white noises $a_{1 t}$ and $a_{2 t}$, denoted as $v=\sqrt{\sigma_{22} / \sigma_{11}}=\sigma_{2} / \sigma_{1}$, is also an important factor affecting the demand planning performance. Given $\underline{\Phi}$ and $v$, the cost reduction functions CR2, CR3, CR4 and CR5 can be then calculated using equations (3)-(8).

However, both $\Phi$ and $v$ cannot be directly observed by demand planners. Alternatively, two statistical properties of demands can be directly observed: the ratio between the standard deviations of actual demands $X_{1 t}$ and $X_{2 t}$, $v_{x 2 x 1}=\sqrt{\sigma_{x 2 x 2} / \sigma_{x 1 x 1}}=\sigma_{x 2} / \sigma_{x 1}$, and the correlation of $X_{1}$ and $X_{2}, \rho=\sigma_{x 1 x 2} / \sigma_{x 1} \sigma_{x 2}$. Given $v$ and $\underline{\Phi}, v_{x 1 \times 2}$ and $\rho$ can be respectively expressed as:

$$
\begin{aligned}
v_{x 2 x 1}= & \left(\left(-\phi_{11}^{3} \phi_{22} v^{2}-\phi_{22} \phi_{21}^{2} \phi_{11}+\phi_{22} \phi_{11} v^{2}+\phi_{11}^{2} v^{2}+\phi_{21} \phi_{11}^{2} \phi_{12} v^{2}+\phi_{21}^{3} \phi_{12}-\phi_{21}^{2}\right.\right. \\
& \left.-v^{2}+v^{2} \phi_{21} \phi_{12}\right) /\left(-\phi_{12}^{2} v^{2}+\phi_{21} \phi_{12}+\phi_{22} \phi_{11}+v^{2} \phi_{21} \phi_{12}^{3}+\phi_{22}^{2}-\phi_{11} \phi_{22}^{3}\right. \\
& \left.\left.-\phi_{22} \phi_{12}^{2} \phi_{11} v^{2}+\phi_{21} \phi_{22}^{2} \phi_{12}-1\right)\right)^{0.5} \\
\rho= & \left(\phi_{21} \phi_{11} \phi_{22}^{2}-\phi_{21}^{2} \phi_{22} \phi_{12}+\phi_{11}^{2} v^{2} \phi_{22} \phi_{12}-\phi_{21} \phi_{11}-v^{2} \phi_{22} \phi_{12}-\phi_{21} \phi_{12}^{2} \phi_{11} v^{2}\right) \\
& \div\left(\left(\phi_{11}^{2}-\phi_{11} \phi_{22}^{3}+\phi_{21} \phi_{22}^{2} \phi_{12}+\phi_{21} \phi_{11}^{2} \phi_{12}+\phi_{11}^{3} \phi_{22}^{3}-\phi_{11}^{3} \phi_{22}-3 \phi_{21} \phi_{11}^{2} \phi_{12} \phi_{22}^{2}\right.\right. \\
& \left.+3 \phi_{21}^{2} \phi_{11} \phi_{12}^{2} \phi_{22}+\phi_{21} \phi_{12}+\phi_{21}^{2} \phi_{12}^{2}+\phi_{22} \phi_{11}-1-\phi_{21}^{3} \phi_{12}^{3}+\phi_{22}^{2}-\phi_{11}^{2} \phi_{22}^{2}\right) \\
& \times\left(\left(\left(\phi_{12}^{2} v^{2}-\phi_{21} \phi_{12}-\phi_{22} \phi_{11}-v^{2} \phi_{21} \phi_{12}^{3}-\phi_{22}^{2}+\phi_{11} \phi_{22}^{3}+\phi_{22} \phi_{12}^{2} \phi_{11} v^{2}\right.\right.\right. \\
& \left.-\phi_{21} \phi_{22}^{2} \phi_{12}+1\right)\left(\phi_{11}^{3} \phi_{22} v^{2}+\phi_{22} \phi_{21}^{2} \phi_{11}-\phi_{22} \phi_{11} v^{2}-\phi_{11}^{2} v^{2}-\phi_{21} \phi_{11}^{2} \phi_{12} v^{2}\right. \\
& \left.\left.-\phi_{21}^{3} \phi_{12}+\phi_{21}^{2}+v^{2}-v^{2} \phi_{21} \phi_{12}\right)\right) /\left(\phi_{11}^{2}-\phi_{11} \phi_{22}^{3}+\phi_{21} \phi_{22}^{2} \phi_{12}+\phi_{21} \phi_{11}^{2} \phi_{12}\right. \\
& +\phi_{11}^{3} \phi_{22}^{3}-\phi_{11}^{3} \phi_{22}-3 \phi_{21} \phi_{11}^{2} \phi_{12} \phi_{22}^{2}+3 \phi_{21}^{2} \phi_{11} \phi_{12}^{2} \phi_{22} \\
& \left.\left.\left.+\phi_{21} \phi_{12}+\phi_{21}^{2} \phi_{12}^{2}+\phi_{22} \phi_{11}-1-\phi_{21}^{3} \phi_{12}^{3}+\phi_{22}^{2}-\phi_{11}^{2} \phi_{22}^{2}\right)^{2}\right)^{0.5}\right)
\end{aligned}
$$

Now, we can evaluate the performance of the demand planning approaches under the eight scenarios and varied values of $v$. For each scenario, say Scenario 3, we first show how a approach, say Approach 2, performs in terms of the cost reduction, denoted as CR2_3, with various $v$. The comparison can be now carried out and interpreted with two observable statistical properties: $v_{x 1 \times 2}$ and $\rho$. For each scenario, three figures will be shown. The first is the cost reduction $(\mathrm{CR} \%)$ performance comparison among Approaches 2-5. The rest of two figures illustrate how the two statistical properties vary with $v$. These two figures will help us explain and compare the approach performances.

Under Scenario 1 (figure 2), the auto-correlation elements $\left(\phi_{11}\right.$ and $\left.\phi_{22}\right)$ and intercorrelation elements $\left(\phi_{12}\right.$ and $\left.\phi_{21}\right)$ are all positive. Approach 2 performs poorly because it lacks statistical forecasting and is unable to capture the time series auto-correlation for more accurate demand plans. However, with aggregation, Approach 2 outperforms approaches without aggregation (Approaches 3 and 5) when the correlation of two time series reaches the lowest $(\rho \rightarrow 0.32)$ in figure 2(b) and the variation sizes become close $\left(v_{x 1 \times 2} \rightarrow 1\right)$ in figure 2(c). Approach 3 also performs poorly because it lacks the capability to capture the interrelation of two demands. With the most accurate time-series model, Approach 5's performance is still poorer than Approach 4, where aggregation is performed and only a not exactly 
(a)

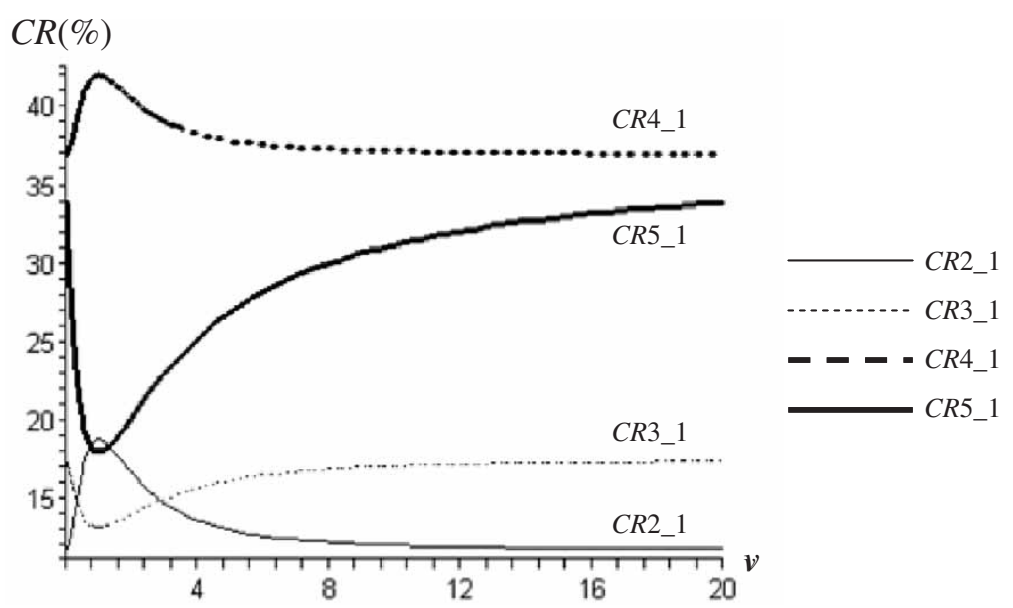

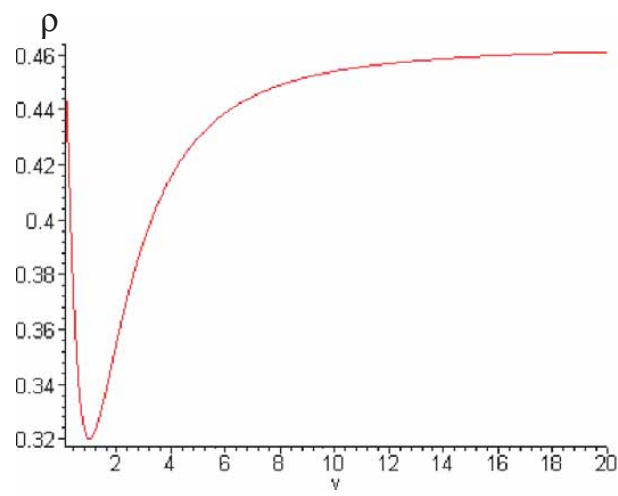

(b)

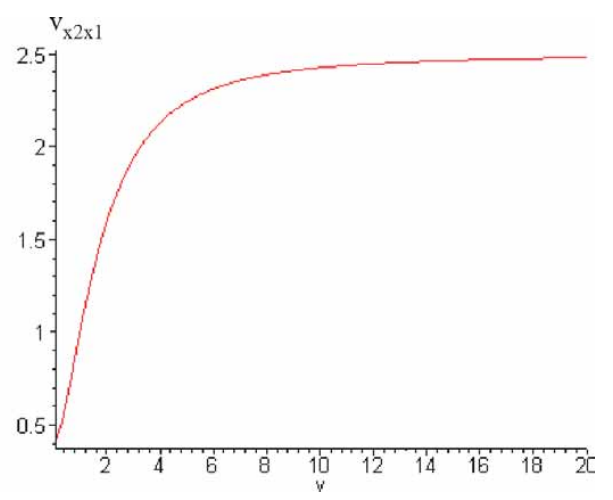

(c)

Figure 2. (a) $\mathrm{CR} \%$ performances; (b) $\rho$ versus $v$; and (c) $v_{x 1 \times 2}$ versus $v$ under Scenario 1.

accurate $\operatorname{AR}(1)$ model is used for statistical forecasting. It is interesting to observe that both aggregation approaches (Approaches 2 and 4) perform best when the correlation is low and the variation sizes are similar. It can be also observed that both Approaches 3 and 5 perform better when the demand correlation is higher since the time-series models can explain more variation that is due to correlation.

Under Scenario 2 (figure 3), both the auto-correlation elements $\left(\phi_{11}\right.$ and $\left.\phi_{22}\right)$ are positive but the inter-correlation elements have opposite signs, i.e. $\phi_{12}<0$ and $\phi_{21}>0$. This opposite effect of $\phi_{12}$ and $\phi_{21}$ causes the demand correlation $(\rho)$ to go from positive to negative as $v>1$ (figure 3(b)). Both aggregation approaches (Approaches 2 and 4) perform relatively well because the aggregated demand becomes much less fluctuated due to the offset of the two negatively correlated demands. Again, both the aggregation approaches perform best when the two variation sizes are not too far off, i.e. $v_{x 1 \times 2}$ is around 1 in figure 3(c). Approach 4, capable of statistical forecasting, performs better than Approach 2 only 
(a)

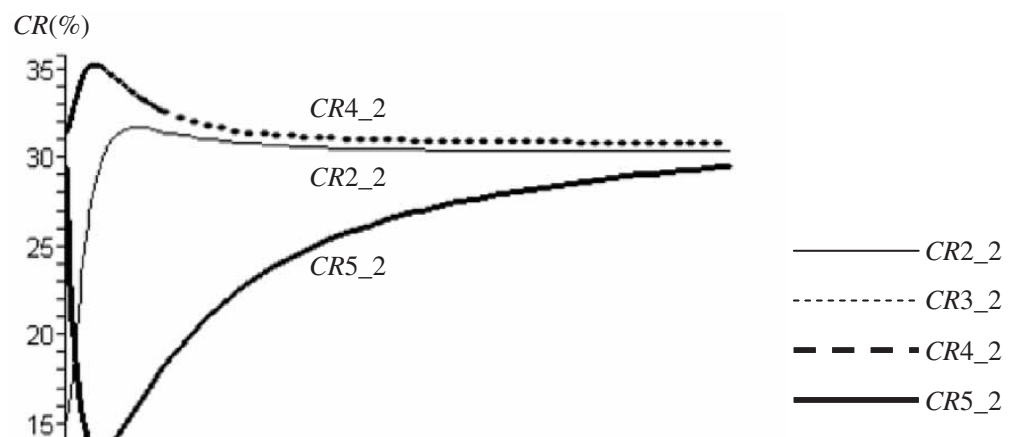

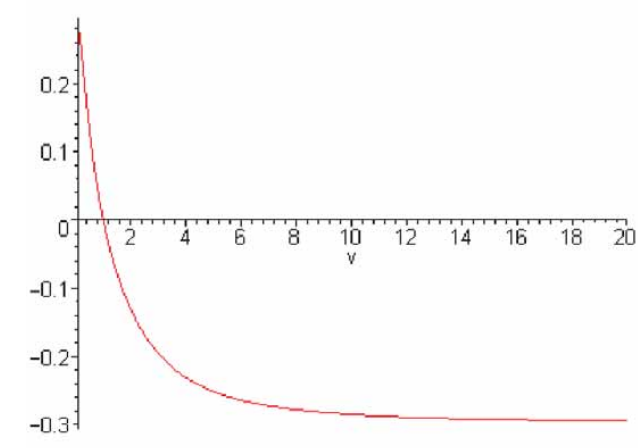

(b)

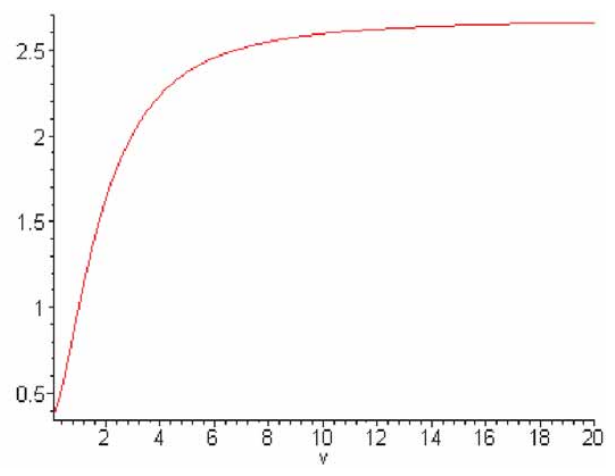

(c)

Figure 3. (a) $\mathrm{CR} \%$ performances; (b) $\rho$ versus $v$; and (c) $v_{x 1 \times 2}$ versus $v$ under Scenario 2.

when the demand correlation is positive and their difference diminishes as the correlation becomes negative. Approaches 3 and 5, with only statistical forecasting capability, perform rather poor. Their performances improve as the demand correlation turns stronger regardless of its sign. With the most accurate time-series model, Approach 5 outperforms Approach 3 expectedly.

It has to be noted that Approach 2 with only simple aggregation could significantly outperform the approaches with sophisticated forecasting functions provided that the correlation is low or negative and its performance approaches Approach 4's when the demand correlation $\rho$ is not higher than -0.15 . This result is rather remarkable and illustrates how effective performing demand aggregation could be.

Similar to Scenario 2, in Scenario 3 (figure 4) both the auto-correlation elements $\left(\phi_{11}\right.$ and $\left.\phi_{22}\right)$ are positive but the inter-correlation elements have opposite signs, $\phi_{12}>0$ and $\phi_{21}<0$. This time, the opposite effect of $\phi_{12}$ and $\phi_{21}$ brings the demand 
(a) $\quad C R(\%)$

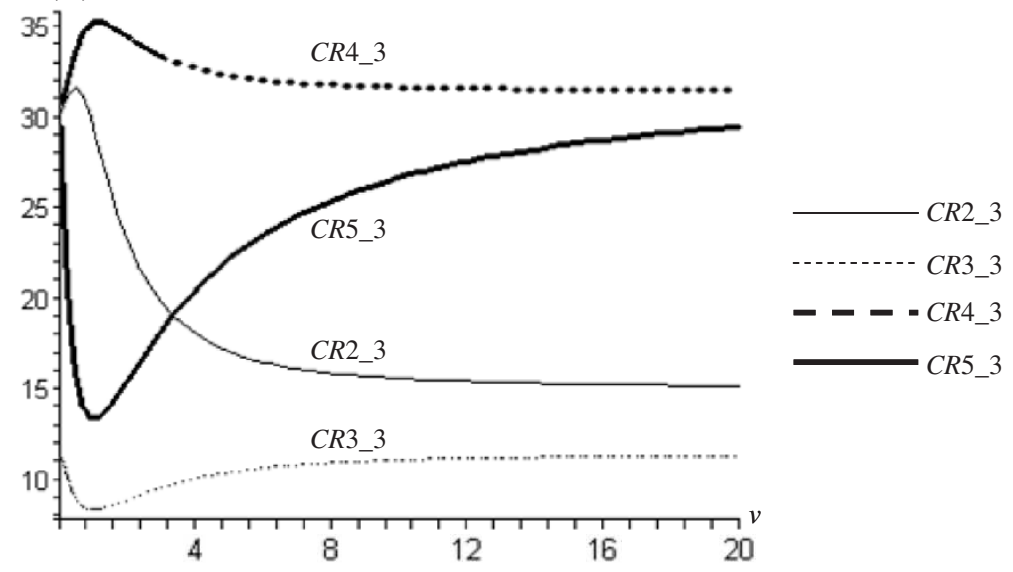

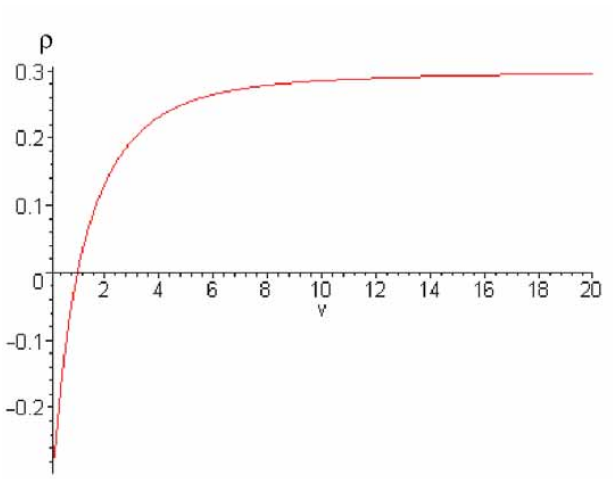

(b)

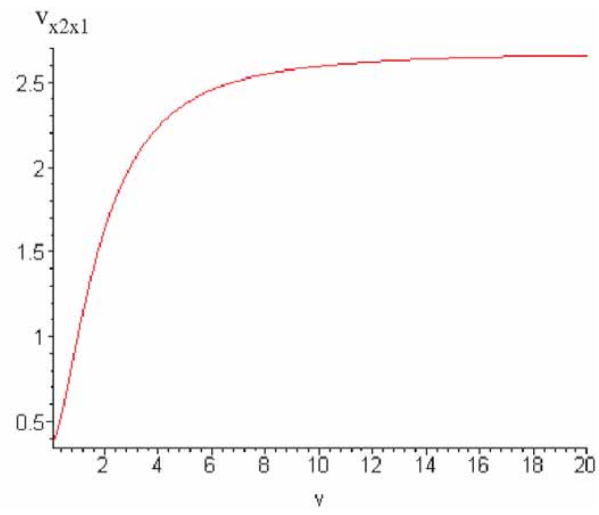

(c)

Figure 4. (a) CR \% performances; (b) $\rho$ versus $v$; and (c) $v_{x 1 \times 2}$ versus $v$ under Scenario 3.

correlation ( $\rho$ ) from negative to positive as $v$ increases (figure 4(b)). The performances of Approaches 3, 4 and 5 are similar to that in Scenario 2. The performance of the simple aggregation approach (Approach 2) becomes poorer as $\rho$ tays positive and becomes larger after $v=1$. However, Approach 2 still outperforms Approach 3. It even outperforms Approach 5, the most sophisticated statistical forecasting approach, when $\rho$ is no higher than 0.2 and $v_{x 1 \times 2}$ is no more than 2. This scenario again illustrates that the aggregation approach is more effective than statistical forecasting approaches.

In Scenario 4 (figure 5), both the intercorrelation elements $\left(\phi_{12}\right.$ and $\left.\phi_{21}\right)$ are positive while the auto-correlation elements have opposite signs, i.e. $\phi_{22}<0$ and $\phi_{11}>0$. This opposite effect of $\phi_{22}$ and $\phi_{11}$ brings the demand correlation $(\rho)$ from positive to negative as in Scenario 2 (figure 5(b)). With no surprise, the performances among four approaches look quite similar to that in Scenario 2. However, the overall cost reduction performance (CR4_4: 28-33\%) is not as good as that 
(a) $C R(\%)$

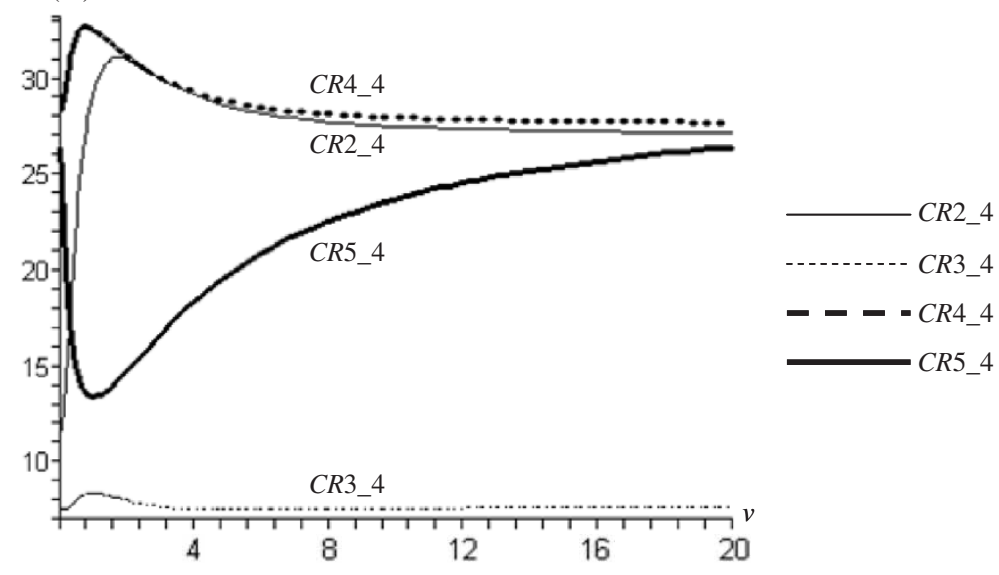

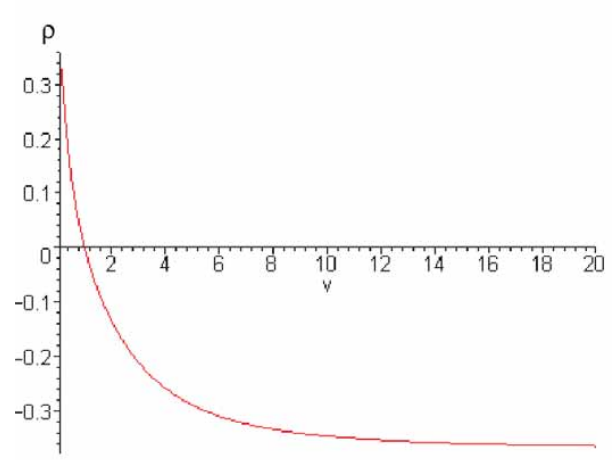

(b)

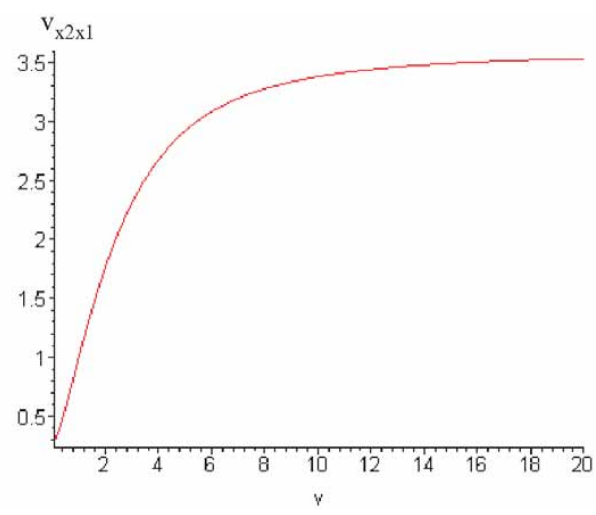

(c)

Figure 5. (a) CR \% performances; (b) $\rho$ versus $v$; and (c) $v_{x 1 \times 2}$ versus $v$ under Scenario 4.

(CR4_2: 33-35\%) in Scenario 2. This is due to the magnitude of $v_{x 1 \times 2}$ grows larger to almost 3.5 in this scenario while it grows only to about 2.6 in Scenario 2. This again confirms that the aggregation is more effective when the variation sizes of the two demands are closer.

Under Scenario 5 (figure 6), the two auto-correlation elements $\left(\phi_{11}\right.$ and $\left.\phi_{22}\right)$ are positive while both the inter-correlation elements $\left(\phi_{12}\right.$ and $\left.\phi_{21}\right)$ are negative. From figure $6(\mathrm{~b})$, the demand correlation appears to be always negative in this scenario. Its degree of correlation becomes weaker from -0.45 to -0.32 as $v$ increases from 0 to 1 and turns stronger again from -0.32 to -0.46 as $v$ increases from 1 to 20 . Thus, Approaches 3 and 5 perform not as good as Approaches 2 and 4 because of the strong negative correlation. As observed in previous scenarios, both Approaches 3 and 5 perform better when the degree of correlation is stronger due to their capability to explain the correlation by time series forecasting. However, Approaches 2 and 4 perform poorer as the demands become more negatively correlated. As in Scenario 2, 
(a) $C R(\%)$

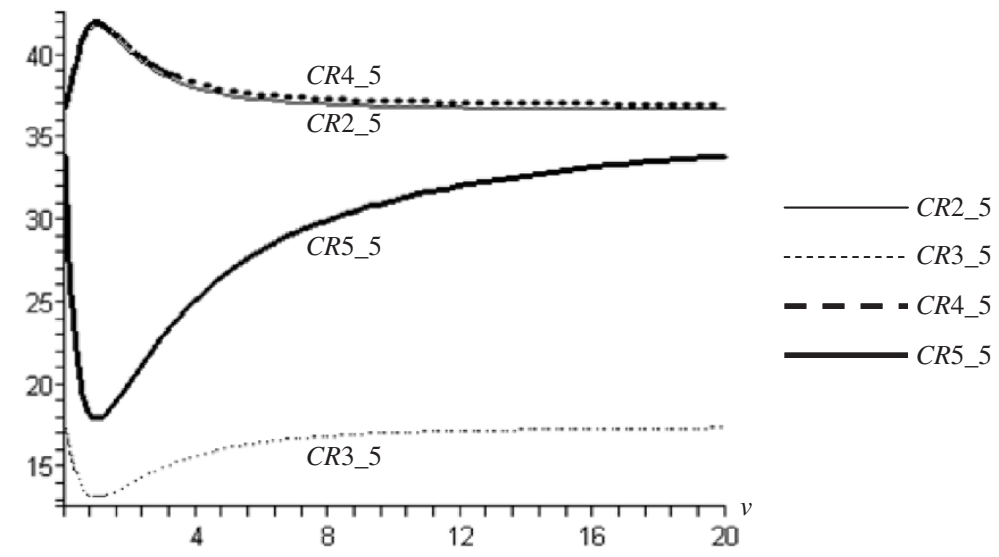

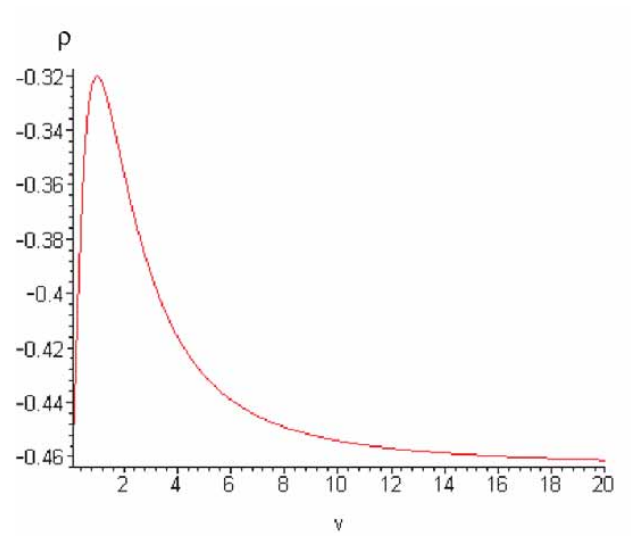

(b)

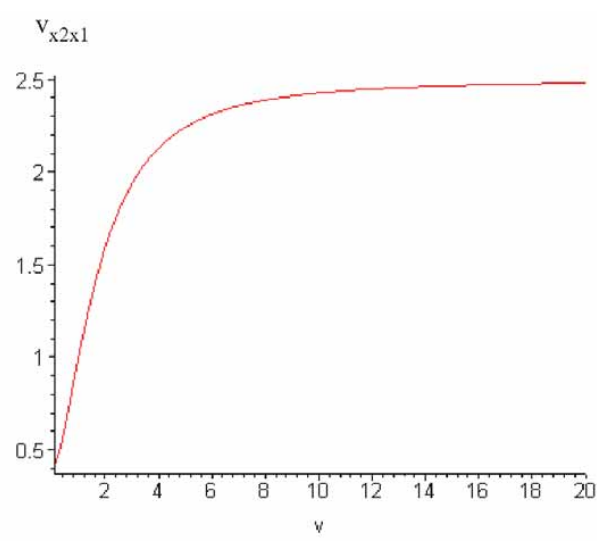

(c)

Figure 6. (a) CR\% performances; (b) $\rho$ versus $v$; and (c) $v_{x 1 \times 2}$ versus $v$ under Scenario 5.

the effect of $v_{x 1 \times 2}$ becomes more important when $\rho$ reaches certain negative level. It can be observed that both the aggregation approaches perform best as the variation sizes come close to each other. That is, the setoff effect by two negatively correlated demands is enhanced by equal variation sizes.

Under Scenario 6 (figure 7), the two auto-correlation elements have opposite signs $\left(\phi_{11}>0\right.$ and $\left.\phi_{22}<0\right)$ and the two inter-correlation elements have two opposite signs $\left(\phi_{12}<0\right.$ and $\left.\phi_{21}>0\right)$. In this scenario, the demand correlation appears to be always positive. Its degree of correlation becomes weaker from 0.34 to 0.19 as $v$ increases from 0 to 1 and turns stronger again from 0.19 to 0.36 as $v$ increases from 1 to 20. This looks very similar to Scenario 1. Thus, Approaches 2, 3 and 5 also perform quite similarly to their performances in Scenario 1. Approach 2 performs relatively well against Approach 3 in this scenario. This is no surprise if one observes that in Scenario 1 Approach 2 already outperforms Approach 3 when the demand 
(a) $C R(\%)$

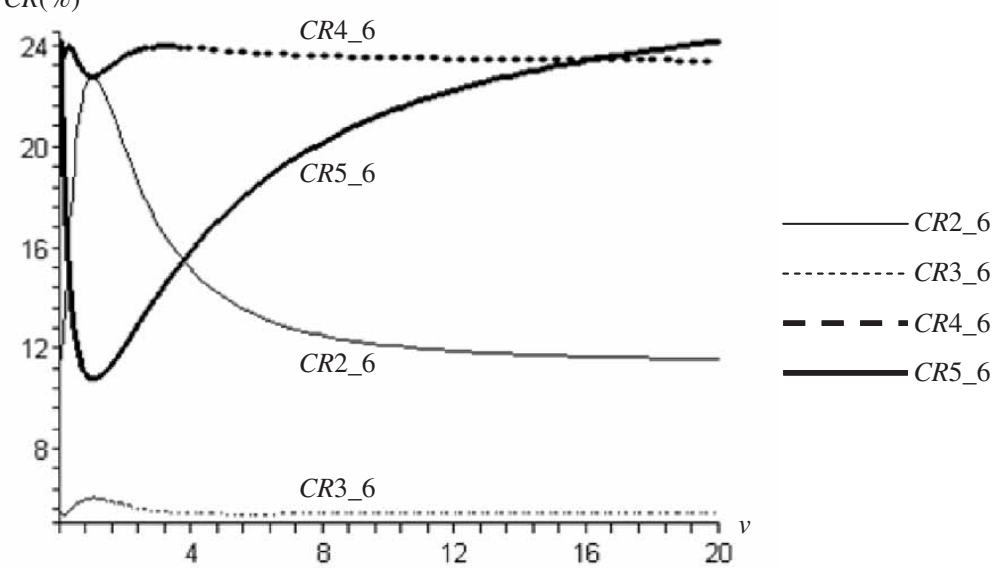

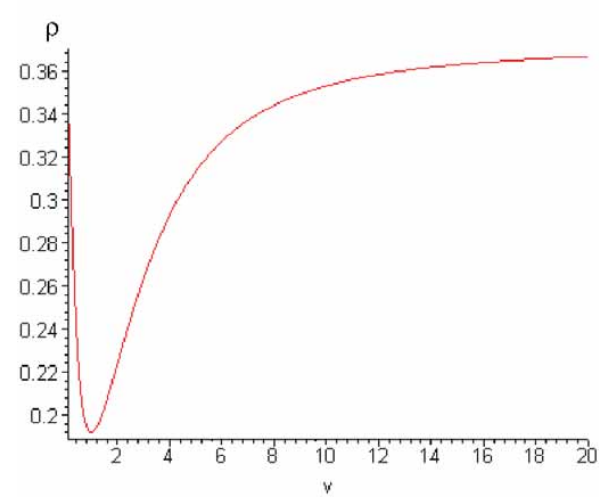

(b)

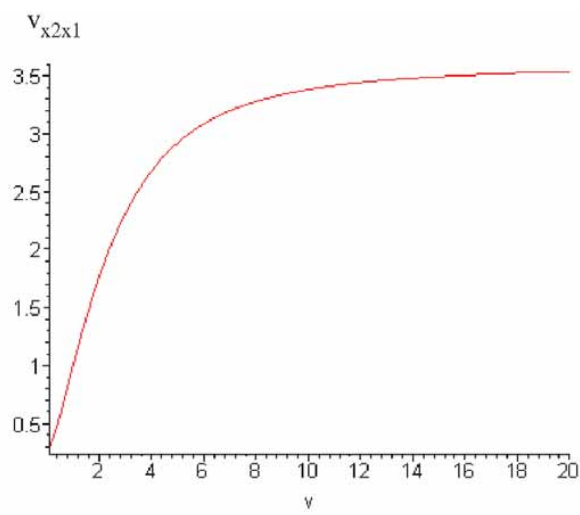

(c)

Figure 7. (a) CR \% performances; (b) $\rho$ versus $v$; and (c) $v_{x 1 \times 2}$ versus $v$ under Scenario 6.

correlation is no higher than 0.38 and outperforms Approach 5 when the demand correlation is lower than 0.32 . It is also interesting to note that the statistical forecasting capability of Approach 4 becomes more significant in this case, where demand correlation is positive but low. Unlike its performance in other Scenarios, Approach 4 behaves more like Approaches 3 and 5 to reach its poorest performance when the demand correlation is the lowest.

Like Scenario 6, Scenario 7 (figure 8) has two opposite-signed auto-correlation elements $\left(\phi_{11}>0\right.$ and $\left.\phi_{22}<0\right)$ and two opposite-signed inter-correlation elements $\left(\phi_{12}>0\right.$ and $\left.\phi_{21}<0\right)$. But the demand correlation behaviour looks more like Scenario 5. Therefore, the performances among approaches look almost the same as in Scenario 5. However, the overall cost reduction improvement is not as good because the demands are less negatively correlated and the difference between variation sizes is greater $\left(v_{x 1 \times 2}\right.$ approaches 3.5 .) The ratio of variation sizes again 
(a) $C R(\%)$

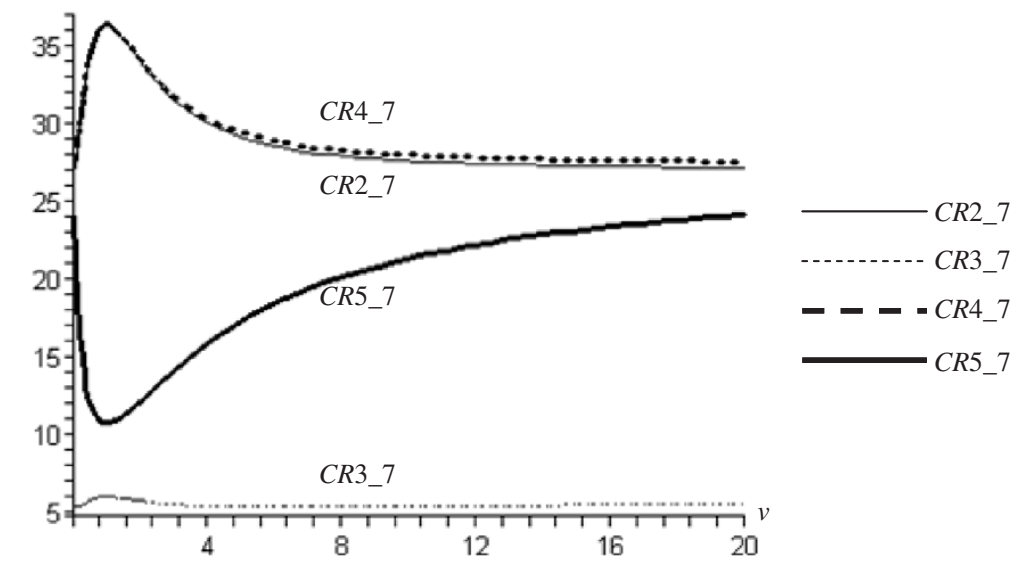

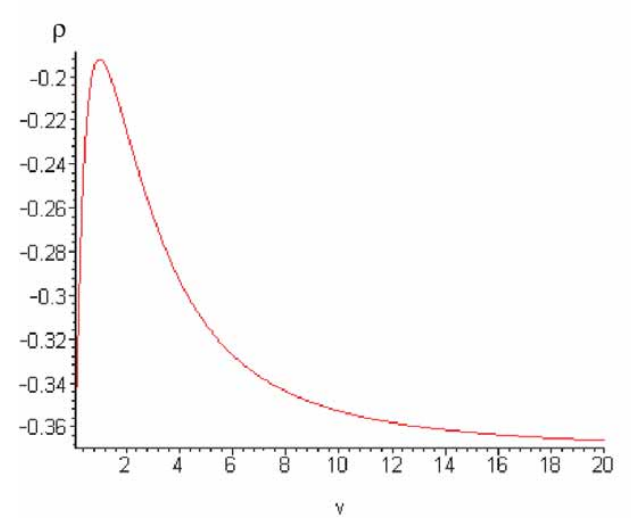

(b)

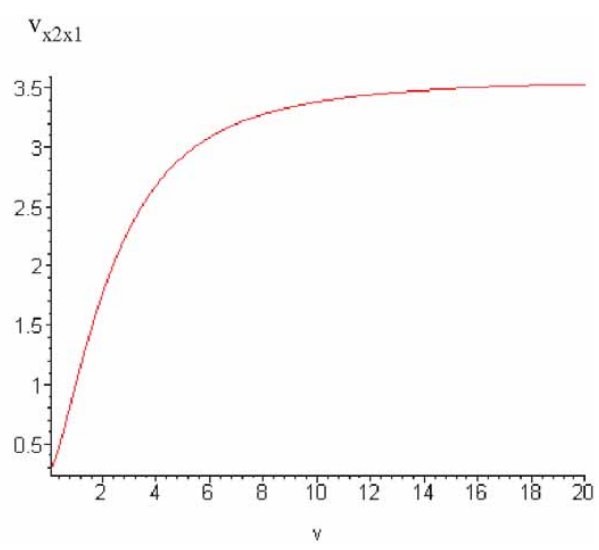

(c)

Figure 8. (a) $\mathrm{CR} \%$ performances; (b) $\rho$ versus $v$; and (c) $v_{x 1 \times 2}$ versus $v$ under Scenario 7.

appears to be the dominating factor for approaches with aggregation (Approaches 2 and 4).

Under Scenario 8 (figure 9), only one auto-correlation element $\left(\phi_{11}\right)$ is positive and the rest of elements are negative. Its demand correlation behaviour is very similar to that in Scenario 3. The performances among approaches are, therefore, alike. Again, the overall cost reduction improvement is poorer in this scenario because the variation sizes are farther off ( $v_{x 1 \times 2}$ approaches 3.5$)$.

\section{Conclusions}

Overall, we have the following observations based on the evaluation and comparison results from the eight scenarios. 
(a) $C R(\%)$

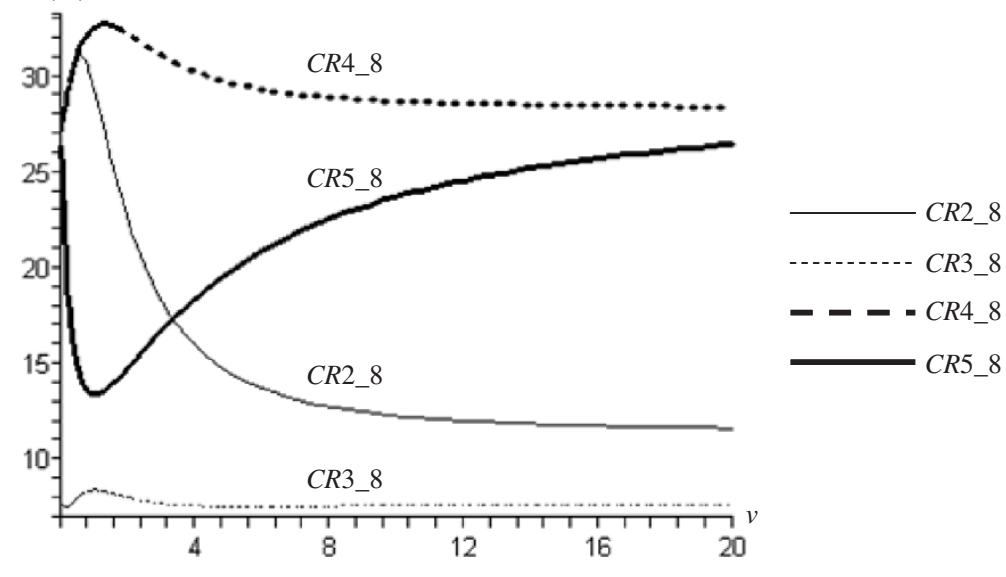

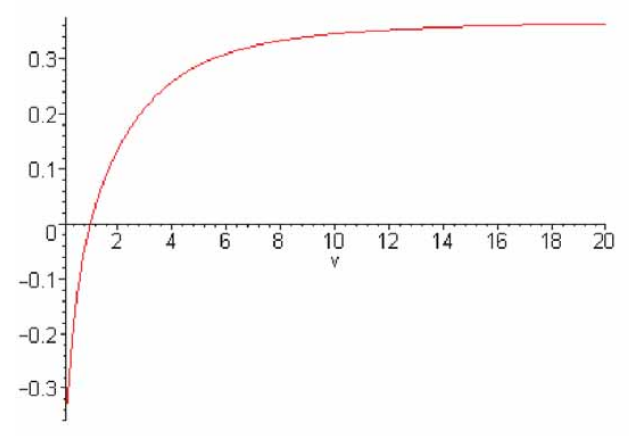

(b)

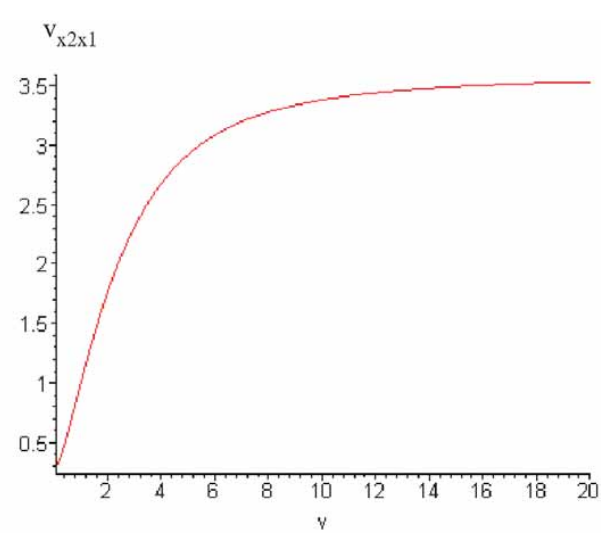

(c)

Figure 9. (a) CR\% performances; (b) $\rho$ versus $v$; and (c) $v_{x 1 \times 2}$ versus $v$ under Scenario 8.

With aggregation and statistical forecasting capabilities, Approach 4 appears to be the best approach regardless of the scenarios. Approach 4's effectiveness is also the most stable and less affected by changes of demand correlation and ratio of variation sizes.

The simple aggregation approach, Approach 2, performs quite as good as Approach 4 and outperforms Approach 5, the most sophisticated statistical forecasting approach, when the demand correlation is weak or negative. Its performance, however, worsens quickly as the demand correlation becomes positive and large and the two variation sizes become significantly different.

Approach 3, even with its statistical forecasting capability, appears to be the worst approach. It outperforms Approach 2 only in Scenario 1 when two demands are more positively correlated and the variations sizes are different. 
Now, we summarize our observations and provide the following principles and guidelines for practitioners to adopt appropriate demand planning approaches under different situations:

- Demand correlation is negative $(\rho<0)$. If aggregating demands only incurs a limited extra cost, Approach 2 appears to be the best choice since it requires only simple aggregation and does not need to build statistical model for forecasting. If demand aggregation will incur a substantial extra cost, Approach 5 should be adopted. However, Approach 5 requires a correct multivariate statistical model for accurate forecasts. When the demand correlation is insignificant but individual demands have significant autocorrelations, Approach 3 is a good choice for making more than $10 \%$ cost reduction.

- Demand correlation is positive $(\rho>0)$. If the correlation is low, $0<\rho<0.2$, and the extra aggregation cost is minimum, Approach 2 is still a good choice given that no statistical model is required for this approach. If the correlation is high, $\rho>0.2$, and the extra aggregation cost is limited, Approach 4 is more preferable. It should be noted that building a univariate time-series model for an aggregated demand in Approach 4 is much more reliable and simpler than building the multivariate time-series model in Approach 5. If the extra aggregation cost is substantially large, Approach 5 has to be adopted. Again, Approach 3 can be used instead when low demand correlation and high auto-correlation are observed.

\section{A.1. Appendix 1: Derivation of $\sigma_{x 1 x 1}, \sigma_{x 2 x 2}$ and $\sigma_{x 1 x 2}$}

With the VAR(1) model $\underline{X}_{t}=\Phi \underline{X}_{t-1}+\underline{a}_{t}$, there exists a cross-covariance matrix of lag 1 , denoted as $\Gamma(l)$, which is defined as:

$$
\begin{aligned}
\Gamma(l) & =\operatorname{Cov}\left(\underline{X}_{t}, \underline{X}_{t-l}\right)=E\left[\left(\underline{X}_{t}-\underline{\mu}\right)\left(\underline{X}_{t-l}-\underline{\mu}\right)^{\prime}\right] \\
& =\left[\begin{array}{ll}
\sigma_{x 1 x 1}(l) & \sigma_{x 1 x 2}(l) \\
\sigma_{x 2 x 1}(l) & \sigma_{x 2 x 2}(l)
\end{array}\right] .
\end{aligned}
$$

This cross-covariance matrix has the following two properties.

Property 1:

For $l=0, \pm 1, \pm 2, \ldots, \Gamma(l)$ satisfies $\Gamma(-l)=\Gamma^{\prime}(l)$.

Property 2:

$$
\Gamma(l)= \begin{cases}\Phi \Gamma(-1)+\Sigma, & l=0 \\ \underline{\Phi^{l}} \Gamma(0), & l \geq 1 .\end{cases}
$$

Property 2 is similar to the Yule-Walker equations in $\operatorname{AR}(1)$ time-series model. 
Variances of two demands, denoted as $\sigma_{x 1 x 1}$ and $\sigma_{x 2 x 2}$, and the covariance, $\sigma_{x 1 x 2}$, could be written in matrix form which equals to the cross-covariance matrix of lag 0 . In Property 1 and 2, we know:

$$
\begin{gathered}
\Gamma(0)=\underline{\Phi} \Gamma(-1)+\Sigma=\underline{\Phi} \Gamma^{\prime}(1)+\Sigma ; \\
\Gamma(1)=\underline{\Phi} \Gamma(0) .
\end{gathered}
$$

Substitute (I.1) into (I.2) to obtain:

$$
\Gamma(0)=\underline{\Phi} \Gamma^{\prime}(0) \underline{\Phi^{\prime}}+\Sigma .
$$

We know $\Gamma(0)=\Gamma^{\prime}(0)$ by Property 2 . Thus, (I.3) becomes

$$
\Gamma(0)=\underline{\Phi} \Gamma(0) \underline{\Phi^{\prime}}+\Sigma .
$$

It is then straightforward to derive $\sigma_{x 1 x 1}, \sigma_{x 2 x 2}, \sigma_{x 1 x 2}$ from (I.4).

\section{A.2. Appendix 2: Proof of Theorem 1}

$\underline{X}_{t}=\underline{\Phi}_{t-1}+\underline{a}_{t}$ follows the bivariate VAR(1) model in (2). Let $\underline{X}_{t}$ and $\underline{a}_{t}$ be zero vectors for $t \leq 0$. That is:

$$
\underline{X}_{t=0}=\underline{\Phi} \underline{X}_{t=-1}+\underline{a}_{t=0}=\underline{a}_{0}=\left[\begin{array}{l}
0 \\
0
\end{array}\right]
$$

Then, (2) becomes:

$$
\begin{aligned}
\underline{X}_{t} & =\underline{\Phi}\left(\Phi X_{t-2}+\underline{a}_{t-1}\right)+\underline{a}_{t} \\
& =\underline{\Phi}^{2}\left(\underline{\Phi X}_{t-3}+\underline{a}_{t-2}\right)+\underline{\Phi a_{t-1}+\underline{a}_{t}} \\
& =\underline{\Phi}^{t} \underline{X}_{0}+\underline{\Phi}^{t-1} \underline{a}_{1}+\underline{\Phi}^{t-2} \underline{a}_{2}+\cdots+\underline{\Phi}^{2} \underline{a}_{t-2}+\underline{\Phi} \underline{a}_{t-1}+\underline{a}_{t} \\
& =\sum_{k=1}^{t} \underline{\Phi}^{k} \underline{a}_{t-k}+\underline{a}_{t}
\end{aligned}
$$

Suppose $\Phi$ has the following eigenvalues and corresponding eigenvectors:

$$
\lambda_{1}, \underline{\mathrm{e}}_{1}=\left[\begin{array}{c}
e_{11} \\
e_{12}
\end{array}\right] ; \quad \lambda_{2}, \underline{\mathrm{e}}_{2}=\left[\begin{array}{c}
e_{21} \\
e_{22}
\end{array}\right] .
$$

Let $\underline{P}=\left[\begin{array}{ll}\underline{e}_{1} & \underline{e}_{2}\end{array}\right]$. Then, $\underline{\Phi}^{k}=\underline{P}^{k} \underline{P}^{-1}$ where

$$
\underline{P}^{-1}=\left[\begin{array}{ll}
\underline{e}_{1} & \underline{e}_{2}
\end{array}\right]^{-1}=\frac{1}{e_{11} e_{22}-e_{12} e_{21}}\left[\begin{array}{cc}
e_{22} & -e_{21} \\
-e_{12} & e_{11}
\end{array}\right] \quad \text { and } \quad \underline{\lambda}=\left[\begin{array}{cc}
\lambda_{1} & 0 \\
0 & \lambda_{2}
\end{array}\right] \text {. }
$$


That is:

$$
\underline{\Phi}^{k}=\frac{1}{e_{11} e_{22}-e_{21} e_{12}}\left[\begin{array}{ll}
e_{11} \lambda_{1}^{k} e_{22}-e_{21} \lambda_{2}^{k} e_{12} & -e_{11} \lambda_{1}^{k} e_{21}+e_{21} \lambda_{2}^{k} e_{11} \\
e_{12} \lambda_{1}^{k} e_{22}-e_{22} \lambda_{2}^{k} e_{12} & -e_{12} \lambda_{1}^{k} e_{21}+e_{22} \lambda_{2}^{k} e_{11}
\end{array}\right]
$$

The bivariate VAR(1) model can be now rewritten as:

$$
\begin{aligned}
{\left[\begin{array}{l}
X_{1 t} \\
X_{2 t}
\end{array}\right]=} & \sum_{k=1}^{t} \frac{1}{e_{11} e_{22}-e_{21} e_{12}}\left[\begin{array}{ll}
e_{11} \lambda{ }_{1}^{k} e_{22}-e_{21} \lambda{ }_{2}^{k} e_{12} & -e_{11} \lambda_{1}^{k} e_{21}+e_{21} \lambda_{2}^{k} e_{11} \\
e_{12} \lambda_{1}^{k} e_{22}-e_{22} \lambda_{2}^{k} e_{12} & -e_{12} \lambda_{1}^{k} e_{21}+e_{22} \lambda_{2}^{k} e_{11}
\end{array}\right]\left[\begin{array}{l}
a_{1 t-k} \\
a_{2 t-k}
\end{array}\right] \\
& +\left[\begin{array}{l}
a_{1 t} \\
a_{2 t}
\end{array}\right] .
\end{aligned}
$$

We can also write the individual equation for $X_{1}$ :

$$
\begin{aligned}
X_{1 t}= & \sum_{k=1}^{t}\left[\lambda_{1}^{k}\left(\frac{e_{11} e_{22}}{C} a_{1 t-k}-\frac{e_{11} e_{21}}{C} a_{2 t-k}\right)\right]+\left(\frac{e_{11} e_{22}}{C} a_{1 t}-\frac{e_{11} e_{21}}{C} a_{2 t}\right) \\
& +\sum_{k=1}^{t}\left[\lambda_{2}^{k}\left(\frac{-e_{21} e_{12}}{C} a_{1 t-k}+\frac{e_{21} e_{11}}{C} a_{2 t-k}\right)\right]+\left(\frac{-e_{21} e_{12}}{C} a_{1 t}+\frac{e_{21} e_{11}}{C} a_{2 t}\right)
\end{aligned}
$$

where $C=e_{11} e_{22}-e_{21} e_{12}$. Therefore, $X_{1 t}$ can be expressed as the sum of $V_{1 t}$ and $V_{2 t}$, where:

$$
\begin{aligned}
& V_{1 t}=\lambda_{1} V_{1 t-1}+a_{1 t}^{v} \quad \text { and } \quad a_{1 t}^{v}=\frac{e_{11} e_{22}}{e_{11} e_{22}-e_{12} e_{21}} a_{1 t}-\frac{e_{11} e_{21}}{e_{11} e_{22}-e_{12} e_{21}} a_{2 t} \\
& V_{2 t}=\lambda_{2} V_{2 t-1}+a_{2 t}^{v} \quad \text { and } \quad a_{2 t}^{v}=\frac{-e_{21} e_{12}}{e_{11} e_{22}-e_{12} e_{21}} a_{1 t}+\frac{e_{21} e_{11}}{e_{11} e_{22}-e_{12} e_{21}} a_{2 t} .
\end{aligned}
$$

Similarly, $X_{2 t}$ can be expressed as the sum of $W_{1 t}$ and $W_{2 t}$ where:

$$
\begin{aligned}
& W_{1 t}=\lambda_{1} W_{1 t-1}+a_{1 t}^{w} \text { and } a_{1 t}^{w}=\frac{e_{12} e_{22}}{e_{11} e_{22}-e_{12} e_{21}} a_{1 t}-\frac{e_{12} e_{21}}{e_{11} e_{22}-e_{12} e_{21}} a_{2 t} \\
& W_{2 t}=\lambda_{2} W_{2 t-1}+a_{2 t}^{w} \text { and } a_{2 t}^{w}=\frac{-e_{22} e_{12}}{e_{11} e_{22}-e_{12} e_{21}} a_{1 t}+\frac{e_{22} e_{11}}{e_{11} e_{22}-e_{12} e_{21}} a_{2 t} .
\end{aligned}
$$

\section{A.3. Appendix 3: Proof of Theorem 2}

Suppose $D_{1 t}$ and $D_{2 t}$ are too stationary AR(1) time series:

$$
\begin{array}{lll}
D_{1 t}=\mu_{d 1}+\varphi_{1} D_{1 t-1}+\varepsilon_{1 t} & \text { and } & \varepsilon_{1 t} \stackrel{\text { i.i.d. }}{\sim} N\left(0, \sigma_{\varepsilon 1}^{2}\right) \\
D_{2 t}=\mu_{d 2}+\varphi_{2} D_{2 t-1}+\varepsilon_{2 t} & \text { and } & \varepsilon_{2 t} \stackrel{\text { i.i.d. }}{\sim} N\left(0, \sigma_{\varepsilon 2}^{2}\right) .
\end{array}
$$


Let $D_{t}=D_{1 t}+D_{2 t} . D_{t}$ is thought to be an AR(1) time series that follows:

$$
D_{t}=\varphi_{a} D_{t-1}+\varepsilon_{t} \text { where } \varepsilon_{t} \stackrel{i . i . d .}{\sim} N\left(0, \sigma_{\varepsilon}^{2}\right) .
$$

Suppose $d_{1 t}, t=1,2,3, \ldots, n$, are $n$ observations of $D_{1 t} ; d_{2 t}, t=1,2,3, \ldots, n$, are $n$ observations of $D_{1 t}$; and $d_{t}=d_{1 t}+d_{2 t} . \phi_{\mathrm{a}}$ and $\sigma_{\varepsilon}^{2}$ are now to be estimated by observations $d_{1 t}$ and $d_{2 t}$ using maximum likelihood estimators (Box et al. 1994):

$$
\hat{\varphi}_{a}=\frac{\sum_{t=2}^{n} d_{t} d_{t-1}}{\sum_{t=1}^{n} d_{t}^{2}} \quad \text { and } \quad \hat{\sigma}_{\varepsilon}^{2}=\frac{\sum_{t=1}^{n} d_{t}^{2}}{n}\left(1-\hat{\varphi}_{a}^{2}\right) .
$$

We can further derive:

$$
\begin{aligned}
\hat{\varphi}_{a} & =\frac{\sum_{t=2}^{n} d_{t} d_{t-1}}{\sum_{t=1}^{n} d_{t}^{2}}=\frac{\sum_{t=2}^{n} d_{1 t} d_{1 t-1}+d_{1 t} d_{2 t-1}+d_{2 t} d_{1 t-1}+d_{2 t} d_{2 t-1}}{\sum_{t=1}^{n} d_{1 t}^{2}+d_{1 t} d_{2 t}+d_{2 t} d_{1 t}+d_{2 t}^{2}} \\
& =\frac{n\left[\hat{\sigma}_{D 1 D 1}(1)+\hat{\sigma}_{D 1 D 2}(1)+\hat{\sigma}_{D 2 D 1}(1)+\hat{\sigma}_{D 2 D 2}(1)\right]+(n-1)\left[\bar{d}_{1}+\bar{d}_{2}\right]^{2}}{n\left[\hat{\sigma}_{D 1 D 1}+2 \hat{\sigma}_{D 1 D 2}+\hat{\sigma}_{D 2 D 2}\right]+n\left[\bar{d}_{1}+\bar{d}_{2}\right]^{2}} .
\end{aligned}
$$

As $n$ approaches infinity, based on the law of large number we obtain:

$$
E\left(\hat{\varphi}_{a}\right)=\frac{\left[\sigma_{D 1 D 1}(1)+\sigma_{D 1 D 2}(1)+\sigma_{D 2 D 1}(1)+\sigma_{D 2 D 2}(1)\right]+\left[u_{D 1}+u_{D 2}\right]^{2}}{\left[\sigma_{D 1 D 1}+2 \sigma_{D 1 D 2}+\sigma_{D 2 D 2}\right]+\left[u_{D 1}+u_{D 2}\right]^{2}}
$$

where:

$\mu_{D 1}$ mean of time series $D_{1 t}$,

$\mu_{D 2}$ mean of time series $D_{2 t}$,

$\sigma_{D 1 D 1}(1)$ covariance of $D_{1 t}$ and $D_{1(t-1)}$ for any $t$,

$\sigma_{D 2 D 2}(1)$ covariance of $D_{2 t}$ and $D_{2(t-1)}$ for any $t$,

$\sigma_{D 1 D 2}(1)$ covariance of $D_{1 t}$ and $D_{2(t-1)}$ for any $t$,

$\sigma_{D 2 D 1}(1)$ covariance of $D_{2 t}$ and $D_{1(t-1)}$ for any $t$,

$\sigma_{D 2 D 1}$ covariance of $D_{2 t}$ and $D_{1 t}$ for any $t$.

Since $\sigma_{D i D i}(1)=\rho_{D i D i}(1) \sigma_{D i D i}(0)$ and $\rho_{D i D i}(1)=\varphi_{i}^{1}$ for $i=1$ and 2 , the above equation becomes:

$$
E\left(\hat{\varphi}_{a}\right)=\frac{\left[\varphi_{1} \sigma_{D 1 D 1}+\sigma_{D 1 D 2}(1)+\sigma_{D 2 D 1}(1)+\varphi_{2} \sigma_{D 2 D 2}\right]+\left[\mu_{D 1}+\mu_{D 2}\right]^{2}}{\left[\sigma_{D 1 D 1}+2 \sigma_{D 1 D 2}+\sigma_{D 2 D 2}\right]+\left[\mu_{D 1}+\mu_{D 2}\right]^{2}} .
$$

Now:

$$
\hat{\sigma}_{\varepsilon}^{2}=\frac{\sum_{t=1}^{n} d_{t}^{2}}{n}\left(1-\hat{\varphi}_{a}^{2}\right)=\left(\left[\hat{\sigma}_{D 1 D 1}+2 \hat{\sigma}_{D 1 D 2}+\hat{\sigma}_{D 2 D 2}\right]+\left[\bar{d}_{1}+\bar{d}_{2}\right]^{2}\right)\left(1-\hat{\varphi}_{a}^{2}\right)
$$

As $n$ approaches infinity, based on the law of large number we obtain:

$$
E\left(\hat{\sigma}_{\varepsilon}^{2}\right)=\left[\sigma_{D 1 D 1}+2 \sigma_{D 1 D 2}+\sigma_{D 2 D 2}+\left(\mu_{D 1}+\mu_{D 2}\right)^{2}\right]\left[1-E\left(\hat{\varphi}_{a}\right)^{2}\right] .
$$




\section{A.4. Appendix 4: Proof of Corollary 1}

Suppose $X_{1 t}$ and $X_{2 t}$ follow bivariate VAR(1) model in (2) and $Y_{t}=X_{1 t}+X_{2 t}$. Then, from Theorem 1, we obtain:

$$
\begin{aligned}
Y_{t}= & \sum_{k=1}^{t}\left[\lambda_{1}^{k}\left(\left(\frac{e_{12}+e_{11}}{C}\right)\left(e_{22} a_{1 t-k}-e_{21} a_{2 t-k}\right)\right)\right]+\left(\left(\frac{e_{12}+e_{11}}{C}\right)\left(e_{22} a_{1 t}-e_{21} a_{2 t}\right)\right) \\
& +\sum_{k=1}^{t}\left[\lambda_{2}^{k}\left(\left(\frac{e_{22}+e_{21}}{C}\right)\left(-e_{12} a_{1 t-k}+e_{11} a_{2 t-k}\right)\right)\right]+\left(\left(\frac{e_{22}+e_{21}}{C}\right)\left(-e_{12} a_{1 t}+e_{11} a_{2 t}\right)\right)
\end{aligned}
$$

Therefore, $Y_{t}$ can be expressed as the sum of $U_{1 t}$ and $U_{2 t}$ where:

$$
\begin{aligned}
& U_{1 t}=\lambda_{1} U_{1 t-1}+a_{1 t}^{u} \quad \text { and } \quad a_{1 t}^{u}=\left(\frac{e_{12}+e_{11}}{e_{11} e_{22}-e_{12} e_{21}}\right)\left(e_{22} a_{1 t}-e_{21} a_{2 t}\right) \\
& U_{2 t}=\lambda_{2} U_{2 t-1}+a_{2 t}^{u} \quad \text { and } \quad a_{2 t}^{u}=\left(\frac{e_{22}+e_{21}}{e_{11} e_{22}-e_{12} e_{21}}\right)\left(-e_{12} a_{1 t}+e_{11} a_{2 t}\right)
\end{aligned}
$$

\section{Acknowledgements}

This research was funded, in part, by SRC-2001-NJ-879 and NSC89-2213E-002-116.

\section{References}

Box, G.E.P., Jenkins, G.M. and Reinsel, G.C., Time Series Analysis Forecasting and Control, 1994 (Prentice Hall: Englewood Cliffs, NJ).

Box, G.E.P. and Tiao, G.C., A canonical analysis of multiple time series. Biometrika, 1977, 64, 355-365.

Caplin, A.S., The variability of aggregate demand with $(s, S)$ inventory policies. Econometrica, 1985, 53, 1395-1409.

Charnes, J.M., Marmorstein, H. and Zinn, W., Safety stock determination with serially correlated demand in a periodic-review inventory system. J. Oper. Res. Soc., 1995, 46, 1006-1013.

Eppen, G.D. and Martin, R.K., Determining safety stock in the presence of stochastic lead time and demand. Manag. Sci., 1988, 34, 1380-1390.

Erkip, N., Hausman, W.H. and Nahmias, S., Optimal centralized ordering policies in multi-echelon inventory systems with correlated demands. Manag. Sci., 1990, 36, 381-392.

Fotopoulos, S., Wang, M.C. and Rao, S.S., Safety stock determination with correlated demands and arbitrary lead times. Eur. J. Oper. Res., 1988, 35, 172-181.

Granger, C.W.J. and Morris, M.J., Time series modelling and interpretation. J. Roy. Stat. Soc. A, 1976, 139, 246-257.

Lau, H.S. and Wang, M.C., Estimating the lead-time demand distribution when the daily demand is non-normal and autocorrelated. Eur. J. Oper. Res., 1987, 29, 60-69.

Lee, H.L., Padmanabhan, V. and Whang, S., Information distortion in a supply chain: bullwhip effect. Manag. Sci., 1997, 43, 546-558. 
Marmorstein, H. and Zinn, W., A conditional effect of autocorrelated demand on safety stock determination. Eur. J. Oper. Res., 1993, 68, 139-142.

Ray, W.D., Computation of reorder levels when the demands are correlated and the lead time random. J. Oper. Res. Soc., 1981, 32, 27-34.

Silver, E.A., Pyke, D.F. and Peterson, R., Inventory Management and Production Planning and Scheduling, 1998 (Wiley: New York).

Simchi-Levi, D., Kaminsky, P. and Simchi-Levi, E., Designing and Managing the Supply Chain, 2000 (McGraw Hill: Boston).

Singh, N. and Nirmalan, T., Identification of composite $(\Sigma+\Pi)$ ARMA models by relatively simpler models. Comm. Stat. Theor. Meth., 1989, 18, 1067-1083.

Tiao, G.C. and Box, G.E.P., Modeling multiple times series with applications. Journal of the American Statistical Association, 1981, 76, 802-816.

Tiao, G.C. and Tsay, R.S., Multiple time-series modeling and extended sample crosscorrelations. Journal of Business and Economic Statistics, 1983, 1, 43-59. 